\title{
The effect of different combinations of boundary conditions on the average radiation efficiency of rectangular plates
}

\author{
G. Squicciarini ${ }^{\text {a }}$ D. J. Thompson ${ }^{\text {a }}$, R. Corradi ${ }^{\mathrm{b}}$ \\ ${ }^{a}$ Institute of Sound and Vibration Research, University of Southampton \\ Southampton, SO17 1BJ, UK \\ ${ }^{\mathrm{b}}$ Politecnico di Milano, Dipartimento di Meccanica \\ Via La Masa 1, Milano, Italy
}

Corresponding author address:

Dr Giacomo Squicciarini

ISVR

University of Southampton

Southampton SO17 1BJ

England

e-mail: g.squicciarini@soton.ac.uk 


\begin{abstract}
The boundary conditions of a vibrating plate are known to have an influence on its sound radiation for frequencies below the critical frequency. To investigate this effect in a systematic way, the average radiation efficiency and radiated power are calculated for a rectangular plate set in an infinite baffle using a modal summation approach. Whereas analytical expressions exist for simply supported boundary conditions, a numerical approach is required for other cases. Nine combinations of boundary conditions are considered, consisting of simply supported, clamped and free edges on different plate edges. The structural vibration is approximated by using independent beam functions in orthogonal directions allowing simple approximate formulae for mode shapes and natural frequencies. This assumption is checked against a finite element model and shown to give reliable results. It is shown that a free plate has the lowest radiation efficiency and a clamped plate the highest for most frequencies between the fundamental panel natural frequency and the critical frequency. Other combinations of boundary condition give intermediate results according to the level of constraint introduced. The differences depend on frequency: excluding the extreme case of a fully free plate all the other boundary conditions give results within a range of $8 \mathrm{~dB}$ in the middle part of the shortcircuiting region, decreasing towards the critical frequency. At low frequency the differences can be even greater, in some cases up to $20 \mathrm{~dB}$. These conclusions are shown to hold for a range of plate thicknesses and dimensions.
\end{abstract}

Keywords: plate vibration, baffled plates, radiation efficiency, radiation ratio, boundary condition.

\title{
1 Introduction
}

In many engineering applications it is important to be able to estimate the noise radiated by a vibrating structure during its design stage. In most cases the structures under consideration, whether industrial machinery, vehicles or civil structures such as bridges, can be subdivided into smaller components; thin vibrating panels, strips and beams are often important components that are 
responsible for noise radiation. To evaluate the noise produced a common procedure is to evaluate the vibration velocity levels of each component and to estimate their acoustic power levels through their dimensions and radiation efficiency. It is particularly useful to study the radiation efficiency of the elementary components in order to be able to characterise the acoustic performance of the structure they form.

The radiation efficiency of an object at a given frequency or frequency band can be defined as the radiated sound power $W_{\text {rad }}$, normalized by the radiating area $S$, the air density $\rho$, the speed of sound $c$ and the space-averaged mean square vibration velocity $\left\langle\overline{v^{2}}\right\rangle$, as:

$$
\sigma=\frac{W_{r a d}}{\rho c S\left\langle\overline{v^{2}}\right\rangle}
$$

The denominator in eq. (1) represents the power that would be radiated by a surface area $S$, vibrating as a rigid piston to produce plane waves, with a mean square velocity equal to the surface-averaged mean-square velocity of the actual object. Furthermore, if the mean square velocity and radiated power are averaged over all possible forcing points over the surface of the structure, the so-called averaged radiation efficiency [1] can be introduced. This is the quantity addressed in this paper.

The radiation efficiency can also be determined for vibration in each single mode, and in this case is referred to as the modal radiation efficiency. This can also be determined as a function of frequency, not just at the natural frequency of the mode in question. Conversely the total radiation efficiency takes into account the effect of all the structural modes according to the excitation at each frequency and is more appropriate for forced vibration. In principle, the radiation efficiency of a plate can be obtained by summing the effect of all modes that contribute significantly in the frequency range under consideration with appropriate weighting.

The calculation of radiation efficiency requires the determination of the radiated power, either the total power or that produced by a single mode. For a flat plate set in an infinite co-planar rigid baffle, the sound pressure can be determined using the Rayleigh integral [2]. There are then two different 
ways used to determine the sound power. The first is to integrate the squared pressure on a hemisphere in the far field; the second is to integrate the acoustic intensity over the vibrating surface. Only the first one is presented and adopted in this work. To calculate the radiation efficiency three different approaches have been adopted in the literature. Some authors have focused on the radiation efficiency of single modes, others have worked on the total radiation efficiency in terms of a summation over the contribution of each mode while a third approach consists in developing asymptotic formulae for the contribution of all modes. The most important findings are reviewed in the following.

Since Rayleigh presented his formulation for assessing the sound radiation from flat finite structures [2], the topic of sound radiation from plates has been widely investigated. The approximation of a flat source set in a co-planar infinite baffle is particularly convenient for studying the main trends, although it is recognised that it differs from the actual situation in practice. Maidanik [3] analysed the acoustic response of panels, including those reinforced via beam systems. Assuming a high modal density of the structures he proposed a simplified asymptotic formulation to calculate the total radiation resistance for simple plates and for ribbed plates.

Wallace [4] used the Rayleigh integral approach to study the power radiated by a single vibration mode of a plate into the far field. This yields a solution for the modal radiation efficiency over the whole frequency range for various mode shapes.

Gomperts investigated the modal radiation efficiency of a flat plate under general boundary conditions. Firstly he proposed a one-dimensional solution for the radiation efficiency of plates [5]. Later [6] he presented results obtained considering a two-dimensional vibration pattern; the plate vibration was determined as the product of the mode shapes of two uniform beams in the two directions. Clear correspondence was found with the results presented by Wallace and in particular it was observed that an increase in the constraints at the edges does not always lead to an increase in the modal radiation efficiency. 
Other approaches to deal with the sound power radiated from planar sources were presented by Heckl [7] in terms of a Fourier Transform in the wavenumber domain and by Williams [8] as a series in ascending power of wavenumber.

Leppington et al. in [9] and [10] introduced several asymptotic formulae to calculate the total radiation efficiency for large acoustic wavenumbers, especially in the range close to the critical frequency. Leppington found that Maidanik's formulae [3] overestimated the radiation resistance at coincidence, particularly for a plate with a very large aspect ratio. The difference in terms of radiation efficiency between a fully simply supported plate and a plate with fully clamped boundaries was found to be around $3 \mathrm{~dB}$ on average below the critical frequency.

In the asymptotic approaches of both Maidanik and Leppington, the radiation efficiency was considered without including the cross-mode contributions. The importance of the cross-modal coupling on the radiation from a panel was pointed out by Keltie and Peng in [11]; it was noted that, particularly at low frequencies and under off-resonant excitation, this contribution should not be neglected.

Snyder and Tanaka [12] demonstrated that the cross-modal contributions are only non-zero for pairs of modes that are either both odd or both even in a given direction. Li and Gibeling [13], [14] found that even at resonances the contribution of mutual radiation may not be insignificant and the total radiated power calculated excluding those terms may under-estimate or over-estimate the correct result.

However, Xie et al. [1], by adopting modal summation approach, demonstrated that in calculating the average radiation efficiency over all possible force positions the contributions from mutual terms are cancelled. They presented the average radiation efficiency of a simply supported plate calculated with a modal summation approach following Wallace's formulation for the modal radiation efficiency. By following the same method and adopting numerical techniques Yoo [15] explored also the effect of 
guided boundary conditions. Putra and Thompson [16] also considered the difference between simply supported and guided boundary conditions for both baffled and unbaffled plates.

In [17] Berry et al. presented a method based on polynomial functions to assess, in a general manner, sound radiation from plates with arbitrary boundary conditions. The modal radiation efficiency was presented along with the total radiation efficiency for simply supported, clamped, guided and free boundary conditions. However, these results have the main limitation that they show radiation efficiency for a plate driven at a single point at the plate centre, in which case many modes of the plate will not be excited. Nevertheless, from these results it can be already concluded that adopting a constant difference of $3 \mathrm{~dB}$ between a clamped and a simply supported plate (as in [10] and [18]) may not be sufficiently accurate.

The method presented by Nelisse et al. [19] also allows different boundary conditions to be taken into account, considering fluid coupling and comparing baffled and unbaffled plates. The radiation efficiency was again calculated for a single point force.

In the work presented by Qiao et al. [20] the structural part of the problem is solved considering the product of beam solutions (as in [6]) in order to obtain the forced response of the plate. The results obtained are interesting considering the purpose of the present work but they are only presented in terms of radiated power and the radiation efficiency is not considered. Similarly, Zhang and Li [21] gave a detailed formulation of the sound radiation from baffled plates with arbitrary boundary conditions by means of a Fourier series. Results were focused on single mode radiation efficiency and on total radiation efficiency for a forcing point at the centre of the plate.

The sensitivity of the radiation efficiency to the location of the forcing point was highlighted by Putra and Thompson in [16]. By comparing the results obtained with twenty different forcing positions they found that the average radiation efficiency lies roughly in the middle of a corridor defined by the $10^{\text {th }}$ and $90^{\text {th }}$ percentile and the width of such a corridor can be up to $20 \mathrm{~dB}$ at low frequency, decreasing 
towards the critical frequency. This corresponds to the effect of cross-modal contributions but presented in this way the conclusion is more directly applicable.

It is known that the assumption of a rigid baffle is artificial. Clearly, having a velocity field equal to zero everywhere, apart from the plate surface, makes the problem easier to solve, but this is not always fully representative of a real structure. For this reason, the problem of an unbaffled plate has also been investigated by some authors ([16], [19] and [21-24]). Some semi-analytical methods have been proposed and the effect of boundary conditions on unbaffled plates has also been addressed ([16] and [23]). In particular, results shown in [16] have shown that, in adopting simply supported and/or guided boundary conditions, the variability in radiation efficiency of an unbaffled plate is much lower than the corresponding baffled plate. It was found that, between the fundamental natural frequency and the critical frequency, the radiation efficiency of a plate guided at its edges is 5 to $15 \mathrm{~dB}$ lower than the simply supported plate, when set in a baffle, while the unbaffled guided plate is only 2 to 4 $\mathrm{dB}$ lower than the equivalent simply supported one. Therefore, the baffled plate configuration is more likely to highlight the effect of modifications in boundary conditions. Moreover, the plate set in a rigid baffle can, in many cases, be a good approximation for the actual arrangement in a built-up structure, or at least it can give a good representation of its acoustic behaviour in a certain frequency range.

This paper is devoted to a systematic investigation of the effect of plate boundary conditions on the average radiation efficiency and acoustic power of baffled rectangular plates. Moreover, the sensitivity of the conclusions to changes in the plate dimensions and thickness are also considered. As described above, similar topics have been already presented in recent years, yet the literature still lacks a complete set of results for total average radiation efficiency with different boundary conditions, especially where the different sides of a plate have different boundary conditions. Analytical solutions are only available in a few cases and in others a numerical solution is required, thus leading to large computational time. In this paper simply supported (S), clamped (C) and free (F) boundary conditions are considered in various combinations to give a total of 9 cases ranging from a 
fully clamped to a free plate. Results are also presented for plates with different aspect ratios and thicknesses for the cases of simply supported, clamped and free boundaries on all four sides.

The methodology presented by Xie et al. [1] for simply supported plates, which used an analytical formula for the modal radiation efficiency [4], is here extended through full numerical computation to consider any set of natural modes. Fluid loading is not taken into account. Therefore the results presented below do not hold for a case of heavy fluid loading or where accurate calculations including fluid loading effects are necessary. Section 2 summarises the methodology as already derived in [1], while Section 3 presents average radiation efficiency and acoustic power results for a variety of cases. The sensitivity to plate geometry is considered in Section 4 .

\section{Radiation efficiency calculation with a modal summation approach}

The equations for calculating the average radiation efficiency using a modal approach were derived in [1] for the case of a simply supported plate. This section summarises the procedure for arbitrary boundary conditions. A numerical integration is then adopted to obtain the results.

\subsection{Plate free vibration}

Consider the plate sketched in Figure 1 with dimensions $a$ and $b$ in the $x$ and $y$ directions, respectively, and thickness $h$. Its vibration is modelled according to Kirchhoff's thin plate theory (see e.g. [26]):

$$
B \nabla^{4} w+\rho_{s} h \frac{\partial^{2} w}{\partial t^{2}}=0
$$

where $B$ is the bending stiffness of the plate, $\rho_{s}$ is the density and $w$ is the plate displacement in the direction perpendicular to the $x-y$ plane. Harmonic motion at circular frequency $\omega$ is assumed with an implicit time dependence of $\mathrm{e}^{\mathrm{j} \omega t}$. Various boundary conditions are included by following solutions presented by Warburton (see [26] and [27]) where the mode shape $\varphi_{m n}(x, y)$ is obtained as the product of beam functions in the $x$ and $y$ directions as 
$\varphi_{m n}(x, y) \cong X(x) Y(y)$

By following the notation used by Leissa [26] (i.e. indices $m$ and $n$ represent the number of nodal lines on the plate), Table 1 summarises the beam functions in the $x$ direction for the boundary conditions adopted; similarly, the beam functions in the $y$ direction can be obtained by replacing $x$ with $y, m$ with $n$ and $a$ with $b$.

The natural frequencies of the plate can be approximated by:

$\omega_{m n}^{2}=\frac{\pi^{4} B}{a^{4} \rho_{s} h}\left\{G_{x}^{4}+G_{y}^{4}\left(\frac{a}{b}\right)^{4}+2\left(\frac{a}{b}\right)^{2}\left[v H_{x} H_{y}+(1-v) J_{x} J_{y}\right]\right\}$

where $v$ is the Poisson's ratio and the coefficients $G, H$ and $J$ are functions related to the boundary conditions and to the order $(m, n)$ of the mode; they are presented e.g. in [26].

The modal mass $M_{m n}$ of the plate is obtained as an integral of the mode shape and mass per unit area over the plate surface $S=a b$ :

$M_{m n}=\int_{S} \rho_{s} h \varphi_{m n}^{2}(x, y) \mathrm{d} S=\rho_{s} h \int_{0}^{a} X^{2}(x) d x \int_{0}^{b} Y^{2}(y) d y$

It is important to note that the solutions presented by Warburton in [27], particularly for cases including free edges, are valid in an approximate sense as already evaluated in [28]; therefore the same approximation holds throughout the present work. The effect of these assumptions on the radiation efficiency is addressed in Section 3.2.

\subsection{Plate response}

Considering a harmonic point force at $\left(x_{0}, y_{0}\right)$ and circular frequency $\omega$, the modal velocity amplitude of the plate is given by:

$u_{m n}\left(x_{0}, y_{0}\right)=\frac{j \omega F \varphi_{m n}\left(x_{0}, y_{0}\right)}{\left[\omega_{m n}^{2}(1+j \eta)-\omega^{2}\right] M_{m n}}$ 
where $F$ is the force amplitude and $\eta$ is the damping loss factor. The plate velocity amplitude can then be obtained by modal summation as:

$v(x, y)=\sum_{m=0}^{\infty} \sum_{n=0}^{\infty} u_{m n}\left(x_{0}, y_{0}\right) \varphi_{m n}(x, y)=\sum_{m=0}^{\infty} \sum_{n=0}^{\infty} v_{m n}$

where $v_{m n}$ represents the contribution of mode $(m, n)$ to the total velocity. Note that the two indices $m$ and $n$ start from 0 to include the case of a fully free plate, in practice the first two indices of the summation depend on the boundary condition considered; for example they both start from 2 in case of a clamped plate at four edges (see Table 1). If the average over all possible forcing positions is introduced, the spatially averaged mean square velocity averaged over all force positions can be formulated as [1]:

$\overline{\left\langle\overline{v^{2}}\right\rangle}=\sum_{m=0}^{\infty} \sum_{n=0}^{\infty} \frac{1}{a b} \int_{0}^{a} \int_{0}^{b} \frac{1}{2}\left|v_{m n}\right|^{2} \mathrm{~d} x_{0} \mathrm{~d} y_{0}=\sum_{m=0}^{\infty} \sum_{n=0}^{\infty} \frac{1}{2} \overline{\left\langle\left|v_{m n}\right|^{2}\right\rangle}$

\subsection{Average radiated acoustic power}

For a plate set in a rigid baffle under harmonic excitation the total acoustic power can be calculated by integrating the acoustic intensity over a hemisphere in the far field as:

$W=\int_{0}^{2 \pi} \int_{0}^{\pi / 2} \frac{|p(\mathbf{r})|^{2}}{2 \rho c} r^{2} \sin \theta \mathrm{d} \theta d \phi$

where $\rho$ is the air density, $c$ is the speed of sound and $p(\mathbf{r})$ is the complex acoustic pressure amplitude at $\mathbf{r}=(r, \theta, \phi)$. By adopting the Rayleigh integral [1],[2] the pressure can be expressed in terms of the plate surface complex velocity $v(x, y)$ as:

$p(\mathbf{r})=\frac{j k \rho c}{2 \pi} \int_{S} v(x, y) \frac{\mathrm{e}^{-j k r^{\prime}}}{r^{\prime}} \mathrm{d} x \mathrm{~d} y$

where $k$ is the acoustic wavenumber and $r^{\prime}=|\mathbf{r}-\mathbf{x}|$ represents the distance between a point $\mathbf{x}$ on the plate surface and a point on the hemisphere at $\mathbf{r}=(r, \theta, \phi)$. 
Combining eq. (10) with eq. (7), the pressure can be given as:

$p(\mathbf{r})=\sum_{m=0}^{\infty} \sum_{n=0}^{\infty} u_{m n}\left(\mathbf{x}_{\mathbf{0}}\right) A_{m n}(\mathbf{r})$

where the terms $A_{m n}$ are defined as:

$$
A_{m n}(\mathbf{r})=\frac{j k \rho c}{2 \pi} \int_{S} \varphi_{m n}(\mathbf{x}) \frac{\mathrm{e}^{-j k r^{\prime}}}{r^{\prime}} \mathrm{d} \mathbf{x}
$$

For simply supported boundary conditions, as shown in [4], an analytical expression can be derived for the terms $A_{m n}(\mathbf{r})$. However, to allow for general boundary conditions, they are obtained here through a double numerical trapezoidal integration. Substitution of eq. (11) into eq. (9) gives a general expression for the total power radiated by a plate forced at $\left(x_{0}, y_{0}\right)$ [1]:

$$
W=\sum_{m=0}^{\infty} \sum_{n=0}^{\infty} \sum_{m^{\prime}=0}^{\infty} \sum_{n^{\prime}=0}^{\infty} u_{m n} u_{m^{\prime} n^{\prime}}^{*} \int_{0}^{2 \pi} \int_{0}^{\pi / 2} \frac{A_{m n}(\mathbf{r}) A_{m^{\prime} n^{\prime}}^{*}(\mathbf{r})}{2 \rho c} r^{2} \sin \theta \mathrm{d} \theta \mathrm{d} \phi
$$

where $m^{\prime}$ and $n^{\prime}$ adopted in the expression for the conjugate highlight the cross-modal contribution.

By averaging over forcing points distributed over the plate surface, the average acoustic power is found as:

$$
\begin{aligned}
\bar{W} & =\frac{1}{a b} \int_{0}^{a} \int_{0}^{b} W \mathrm{~d} x_{0} \mathrm{~d} y_{0} \\
& =\sum_{m=0}^{\infty} \sum_{n=0}^{\infty} \sum_{m^{\prime}=0}^{\infty} \sum_{n^{\prime}=0}^{\infty}\left\{\frac{1}{a b} \int_{0}^{a} \int_{0}^{b} u_{m n}\left(x_{0}, y_{0}\right) u_{m^{\prime} n^{\prime}}^{*}\left(x_{0}, y_{0}\right) d x_{0} d y_{0} \int_{0}^{2 \pi} \int_{0}^{\pi / 2} \frac{A_{m n}(\mathbf{r}) A_{m n}^{*}(\mathbf{r})}{2 \rho c} r^{2} \sin \theta d \theta d \phi\right\}
\end{aligned}
$$

By introducing eq. (6) into eq. (14) it can be shown that, because of the orthogonality of the modeshapes for a uniform plate, the effect of the averaging is to cancel the cross-modal contributions (further details can be found in [1]) and the average radiated power can be expressed as: 


$$
\begin{array}{r}
\bar{W}=\sum_{m=0}^{\infty} \sum_{n=0}^{\infty} \frac{1}{a b} \int_{0}^{a} \int_{0}^{b} u_{m n} u_{m n}^{*} \mathrm{~d} x_{0} \mathrm{~d} y_{0} \int_{0}^{2 \pi} \int_{0}^{\pi / 2} \frac{A_{m n}(\mathbf{r}) A_{m n}^{*}(\mathbf{r})}{2 \rho c} r^{2} \sin \theta \mathrm{d} \theta \mathrm{d} \phi= \\
=\sum_{m=0}^{\infty} \sum_{n=0}^{\infty} \overline{\left|u_{m n}\right|^{2}} \int_{0}^{2 \pi} \int_{0}^{\pi / 2} \frac{A_{m n}(\mathbf{r}) A_{m n}^{*}(\mathbf{r})}{2 \rho c} r^{2} \sin \theta \mathrm{d} \theta \mathrm{d} \phi=\sum_{m=0}^{\infty} \sum_{n=0}^{\infty} \bar{W}_{m n}
\end{array}
$$

where $\overline{\left|u_{m n}\right|^{2}}$ represents the modal velocity amplitude averaged over all forcing points and $\bar{W}_{m n}$ the power radiated by the $(m, n)^{\text {th }}$ mode, again averaged over all forcing points. As previously stated, the integrals in eq. (15) are evaluated numerically with a trapezoidal scheme.

\subsection{Radiation efficiency}

By combining the mean squared velocity averaged over forcing positions, eq. (8), with the average acoustic power, eq. (14), the average radiation efficiency can be finally calculated as:

$$
\bar{\sigma}=\frac{\bar{W}}{\rho c S \overline{\left.\overline{\left\langle v^{2}\right.}\right\rangle}}
$$

\section{Results for different boundary conditions}

Results are first presented for a plate with the same properties as used in [1] for ease of comparison. Therefore an Aluminium plate is considered with a Young's modulus of $7.1 \times 10^{10} \mathrm{~N} / \mathrm{m}^{2}$ and density of $2700 \mathrm{~kg} / \mathrm{m}^{3}$; its dimensions are $0.5 \times 0.6 \times 0.003 \mathrm{~m}$. For this thickness, the critical frequency, $f_{c}=\left(\rho_{s} h / B\right)^{1 / 2} c^{2} / 2 \pi$, is $4 \mathrm{kHz}$.

To ensure that all the fluctuations which characterise the shape of the radiation efficiency curve are captured (see e.g. [1] and [16]), the problem must be properly discretised. In particular, the frequency resolution should be sufficient to allow the contribution of each mode to be correctly assessed; similarly the spatial resolution on the plate surface plays an important role and should include a minimum number of points per structural wavelength; finally the subdivision of the far-field hemisphere into small areas for integrating the acoustic intensity should be fine enough to capture all the acoustic directivity lobes. After some trial and error a good compromise between computational time 
and accuracy has been found for this case by adopting at least 80 points per decade in frequency, a spatial resolution on the plate of $0.0125 \mathrm{~m}$ and by dividing both the angles of the spherical coordinate system into 120 steps. In all the cases presented in the paper all the modes with natural frequencies up to $10 \mathrm{kHz}$ have been included in the summation.

Table 2 summarises the nine combinations of boundary conditions considered in this paper. The reason for selecting the damping values shown in the table is discussed further in the next section. In the following, to describe the boundary conditions of a plate the capital letters of Table 1 are adopted in such a way that for example 'SSFF' represents a plate having simply supported boundary conditions at $x=0$ and $x=a$, and free boundary conditions at $y=0$ and $y=b$.

\subsection{Damping effect}

The methodology adopted for the radiation efficiency calculation allows investigation of the effect of damping. For low damping both the plate vibration and the radiated power will be strongly frequencydependent. Although this can be avoided by using a higher value of loss factor, for high damping the plate radiation becomes dominated by the near-field around the forcing point and the radiation efficiency tends to rise [29]. In order to identify the damping values such that the radiation efficiency is independent of the damping itself, calculations have been performed for the SSSS case for the following four damping loss factor values: $0.3,0.1,0.03$ and 0.01 . Figure 2 summarises the average radiation efficiency of the example plate for these damping values. It can be observed that the difference in terms of radiation efficiency between a plate having a damping loss factor of 0.03 and 0.01 is very small; therefore in the following $\eta=0.01$ is adopted. Similar calculations have been performed on a fully clamped plate (see Figure 3 ) and in this case a similar dependence on damping has been obtained.

In these figures three characteristic frequency regions can be identified, see also [1] and [18]. Below the fundamental natural frequency the radiation of the baffled plate has a monopole character, with $\sigma$ increasing in proportion to $f^{2}$. At the second natural frequency the radiation efficiency drops to a dip 
as it is dominated by this second mode which has a lower modal radiation efficiency. This is due to the phase difference of the motion on different parts of the plate in this mode which will cause the modal radiation efficiency to increase as $f^{4}$ (i.e. like a dipole). A further series of peaks and dips occur above this frequency in what is known as the short-circuiting region (in this region each mode can radiate either as an equivalent monopole, dipole or quadrupole, depending on whether the modeshapes are odd or even in the two directions). At each frequency the exact value of the total radiation efficiency is therefore the result of a combination of all the single modal radiation efficiencies, weighted according to their corresponding squared vibration amplitudes. The radiation efficiency rises to a peak above unity at the critical frequency, in this case $4 \mathrm{kHz}$, before tending to unity at high frequency.

\subsection{Effect of analytical calculation for plate free vibration}

It was mentioned in Section 2.1 that the solution adopted for the plate free vibration is an approximation [28]. Therefore, before going further, a comparison is given of radiation efficiencies calculated adopting the analytical solution for free vibration from [27] (and summarised in Table 1) and a Finite Element modal basis. The procedure presented in Section 2 can, in fact, be applied to any set of natural modes. The effect of the analytical approximation, both in terms of natural frequencies and mode shapes, on the radiation efficiency can readily be investigated. Two representative cases are chosen, the fully free plate and the clamped one. The free boundary condition is known to be the most critical case for these approximations while the analytical solution for clamped boundary conditions should be less critical. In the finite element model the element size has been set to $0.01 \mathrm{~m}$, ensuring at least 5 nodes per wavelength at the highest frequency. Thin plate elements (implementing Kirchhoff plate theory) have been adopted for consistency with the analytical formulation. All the vibration modes in the range $0-10 \mathrm{kHz}$ have been included in the calculation; this has resulted in 335 modes for the free plate and in 308 modes for the clamped.

Figure $4 \mathrm{a}$ shows the results obtained in terms of average radiation efficiency. For the clamped plate the radiation calculated with the analytical modal basis and with the numerical one are very close to 
each other. For the free plate, the position of several peaks and dips is shifted in frequency slightly due to the approximation in the natural frequency calculation. In Figure $4 \mathrm{~b}$ a comparison is given in terms of mean square velocity for the free boundary condition only, again there is not a perfect correspondence between numerical and analytical results. Nevertheless, the most important features of the radiation efficiency curve and mean square velocity are captured with sufficient accuracy by the simplified analytical model and this will be used in the remainder of this paper.

\subsection{Plates with the same boundary condition on all four sides}

The results presented in Section 3.1 can be used to show clearly the difference between the acoustic behaviour of a simply supported plate and a fully clamped plate. The difference between them is shown in Figure 5. For ease of comparison, the narrow band spectra of Figures 2 and 3 have been converted into one-third octave bands and are expressed in decibels as $10 \log _{10} \bar{\sigma}$ (all the following results are presented in this form). In the figure, each line represents the decibel difference between the radiation efficiency of the two plates for a given loss factor. It is clear that this difference depends on the loss factor, being smaller for higher values of damping. For high values of damping the radiation efficiency is relatively independent of the boundary conditions as the radiation becomes dominated by the region around the forcing point [29]. However, the results for loss factors of 0.01 and 0.03 are very close to one another, confirming that the radiation efficiency is effectively independent of damping for values below approximately 0.03 (for the present plate geometry).

It is noteworthy that at very low frequencies, where the response is dominated by the fundamental mode and the acoustic behaviour can be approximated by an equivalent monopole, the simply supported plate radiates more efficiently than the clamped one. Considering the fundamental mode shape of a clamped plate compared with a simply supported one it is clear that the vibration of the clamped plate is limited to a smaller region towards the centre of the plate due to the higher constraints at the boundaries; the radiation efficiency is smaller for a smaller vibrating object, so that the clamped case will be similar to a smaller SSSS plate. 
Moreover, it can be noted that the difference varies considerably with frequency and can be as high as $10 \mathrm{~dB}$ in the range of transition between the fundamental mode and short-circuiting regions around $100 \mathrm{~Hz}$. In the short-circuiting region the difference ranges between 1 and $4 \mathrm{~dB}$ for the lowest loss factors considered while it is generally less than $1 \mathrm{~dB}$ for $\eta=0.3$. In all the cases the difference drops to $0 \mathrm{~dB}$ at and above the critical frequency as the radiation efficiencies tend to unity. It is clear from these results that the difference between these two boundary conditions is not a constant $3 \mathrm{~dB}$ as suggested in [10] and [18].

The one-third octave band radiation efficiency and radiated sound power for a unit mean-square force are shown in Figure 6 for the three cases of Table 2 in which the four edges have the same type of boundary condition, that is $\mathrm{S}, \mathrm{F}$ or $\mathrm{C}$. The free plate has a much lower radiation efficiency than the other two over much of the frequency range, with differences of up to $20 \mathrm{~dB}$ compared with the clamped plate. As well as the fundamental bending mode at $46 \mathrm{~Hz}$, the free plate has three rigid body modes at $0 \mathrm{~Hz}$ and a twisting mode at $35 \mathrm{~Hz}$.

The acoustic power levels in Figure $6 \mathrm{~b}$ reflect, in this case, the trend of the radiation efficiency lines: a high peak is present at the frequency of the fundamental bending mode for the clamped and simply supported plates while in the region between the fundamental natural frequency and the critical frequency the clamped plate tends to radiate more. However, there are a few frequency bands, immediately above the fundamental natural frequency, where the acoustic power of the simply supported plate is greater than that of the clamped plate (e.g. $3.5 \mathrm{~dB}$ higher at $200 \mathrm{~Hz}$ ). For the free plate, the acoustic power is constant at $-54 \mathrm{~dB}$ up to approximately $500 \mathrm{~Hz}$, where it starts to increase. This is the acoustic power level of a rigid piston having the same mass per unit area as the plate and driven by an oscillating unit force, given by

$W=\frac{\rho}{4 \pi c\left(\rho_{s} h\right)^{2}}$

A derivation of eq. (17) is given in [17] [17] and a similar result for guided boundary conditions is presented [15]. This is shown in Figure $6 \mathrm{~b}$ with a dashed line, indicating that the acoustic power of the 
free plate matches exactly with the rigid piston up to $500 \mathrm{~Hz}$. Further explanations about this behaviour of the free plate are given below.

In general, the comparison among FFFF, SSSS, and CCCC boundary conditions suggests that the radiation efficiency increases, on average, with an increasing amount of constraint, even though at some single frequencies an opposite trend can be found. In particular, in the lower part of the shortcircuiting region a simple ranking of radiation efficiencies with boundary conditions is not always straightforward, for two reasons. Firstly, the natural frequency of the fundamental mode generally varies with boundary conditions, so the lower limit of the short-circuiting region differs case by case. Secondly, the way in which the first few modes after the fundamental one are combined together results in a series of peaks and dips that depend both on the mode shapes (even or odd) and on the exact position of each natural frequency. Both of these factors are affected by the boundary conditions so that the position and amplitude of the peaks/dips is different case by case. As a consequence, in this frequency range, the radiation efficiency curves for different boundary conditions tend to intersect one another several times (see also below with other boundary conditions). In the upper part of the shortcircuiting region, starting approximately from $800-1000 \mathrm{~Hz}$ for this plate geometry, many modes contribute to the radiation efficiency and it is more obvious that the radiation efficiency is larger for greater constraints. However, the differences between the results for different boundary conditions reduce as the frequency approaches the critical frequency.

Before discussing the further combinations of boundary conditions, it is instructive to consider the narrow band acoustic power levels of the free plate and clamped plate, shown in Figure 7. To emphasize the low frequency region, results are shown between 10 and $2000 \mathrm{~Hz}$. The contribution of each mode is plotted in thin lines while the total power is represented with thick black lines. Moreover, the modal power corresponding to the first mode is depicted by a light dashed line. For the free plate this is the power due to the rigid body motion (no nodal lines), while for the clamped plate this is the typical fundamental bending mode with two nodal lines per direction (i.e. the four boundaries). 
The clamped plate has no rigid body modes so its acoustic power (Figure 7b) is characterised by the modal power of the plate bending modes in the whole frequency range.

For the free plate, however, (Figure 7a) the rigid body motion dominates the acoustic response up to $150 \mathrm{~Hz}$. Several flexible plate modes are present in this region but their acoustic power at resonance is 10 to $20 \mathrm{~dB}$ lower than the rigid body contribution, but this is dependent on the damping level adopted. The modal acoustic power is the product of modal squared-velocity, which for the free plate decreases as $f^{2}$ (rigid body motion), and radiation efficiency, increasing as $f^{2}$ (in the monopole region); the result is a constant modal power. At higher frequencies (above $150 \mathrm{~Hz}$ in this case) the modal radiation efficiency tends to unity while the averaged velocity still decreases as $f^{2}$, consequently the acoustic power decreases with the same slope. Between $150 \mathrm{~Hz}$ and $500 \mathrm{~Hz}$ the two rigid rotations also contribute in a significant way to the total power. The modal power related to these two rigid motions is depicted in black in Figure 7a (they can be easily recognised since they do not show any peak). The sum of rigid rotations and rigid translation results in a total power which is still approximately constant up to $500 \mathrm{~Hz}$. The first significant contribution from a non-rigid mode appears, in the narrow band spectrum, at around $400 \mathrm{~Hz}$. Above $500 \mathrm{~Hz}$ the total acoustic response is determined by weighted combinations of flexural modes and the power begins to rise.

However the acoustic power at resonance is affected by damping and it has been verified that lower damping levels increase the acoustic power of flexural resonances. Considering for example the $63 \mathrm{~Hz}$ frequency band (i.e. the one governed by the third flexural mode), if the loss factor is reduced by a factor of 10 (from 0.01 to 0.001 ) the acoustic power level is increased by $1.6 \mathrm{~dB}$. This suggests that for very low damping values the effect of bending modes on the acoustic power at low frequencies can be greater than that shown in Figure 7. Nevertheless, the average radiation efficiency does not change significantly if the loss factor is reduced below 0.01 . 


\subsection{Sound radiation for other boundary conditions}

The one-third octave band radiation efficiency and radiated sound power for a unit mean-square force are now shown here for the remaining six cases listed in Table 2. Figure 8 shows the results for three cases where opposite pairs of edges have the same boundary conditions; the free plate and fully clamped plate, from Figure 6, are still shown with broken lines for reference. The radiation efficiency reduces on average as the level of constraint is reduced, i.e. in the order: clamped, simply supported, free. However, below the fundamental natural frequency the case SSFF has the highest radiation efficiency. The acoustic power results in Figure $8 \mathrm{~b}$ again show the same tendency as the radiation efficiency.

Also in this case it is possible to divide the short-circuiting region into two parts. Between the fundamental mode and around $800-1000 \mathrm{~Hz}$ both the radiation efficiency and acoustic power curves intersect a few times. In general more constrained plates result in more radiation. However, in some single one-third octave bands, less constrained plates can radiate more than more constrained ones. Conversely, in the upper part of the short-circuiting region, the three examples considered can be readily ranked according to their radiation efficiency; starting from the less efficient one obtains: SSFF, CCFF and then SSCC.

Results are presented in Figure 9 for three cases where opposite edges have different boundary conditions. Again the radiation efficiency reduces as the level of constraint is reduced. In these cases the radiation efficiency curves do not intersect anymore above $200 \mathrm{~Hz}$ and the CSCS plate is the most efficient. The contributions of single modes appear in the acoustic power (Figure 9b) up to 800-1000 $\mathrm{Hz}$.

It is also interesting to compare the radiation efficiency for plates with the same type of boundary condition applied to different edges, e.g. FSFS against SSFF. This comparison is shown in Figure 10. The pair F-S is presented in Figure 10a; in the monopole region and in the lower part of the shortcircuiting region there are some significant differences. Above about $500 \mathrm{~Hz}$ the two curves show nearly the same radiation efficiency levels; for a small aspect ratio like the one chosen here where 
many modes are contributing, applying different combinations of boundary conditions seems not to have a significant effect on the average radiation efficiency. A similar trend has been found in the corresponding acoustic power (not shown here). Figure 10b and Figure 10c confirm these conclusions for the other two pairs of boundary condition analysed (C-F and S-C). In particular, results found for the CSCS plate are very similar to those found for the SSCC one at all frequencies.

\subsection{Normalised results}

Focusing on the short-circuiting region, and above, Figure 11 shows the radiation efficiencies of all nine cases. Here, the frequency is normalised to the critical frequency and the radiation efficiency to the semi-empirical results known as the Maidanik formula [3]:

$$
\sigma= \begin{cases}\max \left(\frac{4 \pi^{2}}{c^{2} S} \frac{B}{\rho_{s} h}, \frac{\mathrm{Pc}}{4 \pi^{2} S f_{c}} \frac{\left(1-\alpha^{2}\right) \ln \frac{1+\alpha}{1-\alpha}+2 \alpha}{\left(1-\alpha^{2}\right)^{3 / 2}}\right) & \text { for } f_{1,1}<f<f_{c} \\ 0.45 \sqrt{\frac{P f_{c}}{c}} & \text { for } f \approx f_{c} \\ \left(1-\frac{f_{c}}{f}\right)^{-1 / 2} & \text { for } f>f_{c}\end{cases}
$$

where $P$ is the perimeter of the plate and $\alpha=\left(f / f_{c}\right)^{1 / 2}$. Note that the dependence of the fundamental natural frequency on the boundary conditions would make such a normalisation misleading in the very low frequency range; this region is therefore excluded from the figure. Eq. (18) was originally produced for the modal average radiation of a simply supported lightly damped plate. Figure 10 clearly shows that the difference between the current results and eq. (18) is frequency-dependent and also depends significantly on the boundary constraints. The fully clamped case fluctuates around +4 $\mathrm{dB}$ for $0.07<f / f_{c}<0.25$ and then it gradually decreases towards zero. The two combinations CCSS and CSCS follow a similar trend with results about $1 \mathrm{~dB}$ lower. The SSSS case is about 1-2 dB above Maidanik's formula. The two combinations of free and clamped edges are close to $0 \mathrm{~dB}$, while the combinations of free and simply-supported edges give a radiation efficiency lower than the reference curve; significant fluctuations are still present for $f / f_{c}<0.2$, but above this the difference with respect 
to the reference is between -2 and $-1 \mathrm{~dB}$. The free plate was already shown as having the lowest radiation efficiency; in comparison with the reference formula it fluctuates between -6 and $-8 \mathrm{~dB}$ in the range $f / f_{c}<0.6$, above which it increases towards zero.

In conclusion, within the corner/edge mode region, by decreasing the amount of constraint the radiation efficiency tends to reduce, although, as already observed in [5], there are exceptions. Finally, it is important to note that, when considering a baffled plate, the effect of different boundary conditions is considerable and can increase/decrease the noise by up to $20 \mathrm{~dB}$ in a given frequency band. The effect reduces as the frequency approaches the critical frequency.

\section{$4 \quad$ Results for different plate geometry}

The sensitivity of the above conclusions to changes in plate aspect ratio and thickness are next considered for simply supported, clamped and free boundary conditions, i.e. SSSS, CCCC and FFFF. Tables 3 and 4 summarise the corresponding geometry chosen.

\subsection{Effect of aspect ratio}

The average radiation efficiency and acoustic power are presented here for two more aspect ratios of the plate (2.2 and 3.5; the previous results are for an aspect ratio of 1.2). In each case the dimensions are chosen such that the fundamental natural frequency of the SSSS case is kept roughly constant. The plate thickness remains $3 \mathrm{~mm}$. Table 3 gives details of the plate geometry.

The values of the first four natural frequencies for the SSSS case are also given in Table 3. By modifying the aspect ratio the sequence of modes of the plate is modified. In particular the order in which the number of nodal lines in the two directions appear is changed. The trend of the modal radiation efficiency in the low-mid frequency range is greatly affected by the number of nodal lines since this determines whether the modes radiate as a monopole, dipole or quadrupole. The total radiation efficiency will consequently also be affected.

Figures 12 and 13 show the results, again in terms of average radiation efficiency and acoustic power. The results for aspect ratios 2.2 and 3.5 behave similarly to those for aspect ratio 1.2, shown in Figure 
6. By observing these figures the same characteristics highlighted for aspect ratio 1.2 can be noticed: the simply supported plate has the highest radiation efficiency in the monopole region, while in the short-circuiting region the clamped plate is the most efficient and the free plate the least.

Results for the three aspect ratios together are summarised in Figure 14. As before, the frequency is normalised to the critical frequency and the radiation efficiency to eq. (18). For $f / f_{c}>0.2$ the results for each boundary condition form a clearly well separated set of lines. This suggests that, as soon as a large enough number of modes contributes to the response, the effect of the aspect ratio becomes less important.

\subsection{Effect of thickness}

Another geometrical parameter that can be important for the acoustic properties of the plate is the thickness. In particular, an increase in thickness will result in an increase in stiffness and mass of the plate; the natural frequencies of each mode will increase in proportion to $h$. Moreover, thicker plates will have a lower critical frequency. To understand the effect that thickness has on the average radiation efficiency and on the acoustic radiated power, the examples of Table 4 have been considered for SSSS, FFFF and CCCC boundary conditions.

Figures 15, 16 and 17 give the results for each boundary condition separately. Note that the lines corresponding to a thickness of $3 \mathrm{~mm}$ are taken from Figure 6 . Because of the different values of both the fundamental natural frequency and the critical frequency, the short-circuiting region covers different ranges. For this reason, when observing the results, it must be kept in mind that between plates having different thickness there is an overlap between the monopole region and the shortcircuiting one which, at a given value of frequency, leads to very large differences in radiation efficiencies.

Below the fundamental bending natural frequency of the thinner plate, the three plates radiate with the same efficiency and this applies to each boundary condition analysed but, in each case, since the thinner plate has a greater vibration velocity, its radiated powers show higher values. 
The variation of acoustic power with thickness for the FFFF plate is clearly visible in Figure 15b: there is an increase of about 3-4 $\mathrm{dB}$ (see also eq. (17)) for the decrease in thickness presented and the difference among plates with different thickness is nearly constant up to $400 \mathrm{~Hz}$. In this sense Figure $15 \mathrm{~b}$ suggests that, for a given mass per unit area, the acoustic power is nearly independent of frequency up to $f / f_{c}$ of $0.1-0.2$. However the small fluctuations shown for the thicker plate also imply that for thicker plates the acoustic contribution of bending modes can be, at least at their resonance, of similar importance as the rigid body modes. In particular, narrow band results (not presented here) have shown that the $5^{\text {th }}, 8^{\text {th }}$ and $10^{\text {th }}$ modes, at their respective natural frequencies, have an acoustic power comparable to or greater than the rigid body motion. Also in this case lower damping levels would increase the contribution of flexural modes with respect to the rigid motion.

For the three boundary conditions shown, in the short-circuiting region, the radiation efficiency increases with increasing thickness, apart from a few frequency bands at low frequency. A larger radiation efficiency does not imply that, for a given force, the plate is also characterised by a greater acoustic power; the acoustic power of a thinner plate tends to be higher also in the region where its radiation efficiency is lower, due to the higher vibration velocity levels of the thinner plates.

Finally, the results of Figures 15, 16 and 17 are summarised in Figure 18. Normalisation to the critical frequency and to eq. (17) is again applied. Three different sets of lines can again be readily distinguished, one for each type of boundary condition. Within the same set, the difference among different thicknesses is small for $f / f_{c}>0.2$. The three lines for a clamped plate drop from $+4 \mathrm{~dB}$ to 0 in the range $0.2<f / f_{c}<1$. The results for the simply supported plate show a difference with the reference formula of approximately $1 \mathrm{~dB}$ before dropping to zero at the critical frequency. The free plate shows a difference with the reference that ranges between -6 and $-10 \mathrm{~dB}$ for $f / f_{c}<0.5$ before tending to zero. Also for this case it can be observed that, when the number of modes involved in the response is high enough, the radiation efficiency, with frequency normalised to the critical frequency, is largely independent of the thickness of the plate. 


\section{Conclusions}

The average radiation efficiency of a baffled rectangular plate has been calculated for simply supported edges, clamped edges, free edges and various combinations of these.

For the simply supported and the fully clamped examples four different values of loss factor have been considered. It has been found that below $\eta=0.03$ the radiation efficiency is largely independent of damping whereas for high values of damping the radiation efficiency is relatively independent of the boundary conditions as the radiation becomes dominated by the region around the forcing point.

A total of nine combinations of boundary conditions have been considered. It has been found that the free plate generally has the lowest radiation efficiency, while the fully clamped plate is the most efficient at nearly all frequencies above the fundamental mode region. Below the fundamental natural frequency the behaviour is somewhat reversed and the simply supported plate has the greatest radiation efficiency. At and above the critical frequency the boundary conditions are no longer important and the radiation efficiencies all tend to unity. In general the effect of modifying the boundary conditions is considerable and can lead to a large (up to $20 \mathrm{~dB}$ in a given frequency band) increase/reduction in radiated power. In the short-circuiting region, away from the fundamental mode, increasing constraint generally gives an increase in radiation efficiency but the differences are frequency-dependent, reducing as the frequency approaches the critical frequency.

The three most representative combinations of boundary condition (i.e. fully free, clamped and simply supported) have been tested against changes in aspect ratio and thickness. The variability introduced with aspect ratio is not large enough to expect that the conclusions about the effect of boundary conditions drawn are significantly affected by plate dimensions.

Thickness is an important parameter for the radiation efficiency in the short-circuiting region. Increasing thickness from $2 \mathrm{~mm}$ to $3 \mathrm{~mm}$ results in an increase of 3-4 dB and a further increase to 5 mm gives an additional 4-5 dB. However, this is found for all the three boundary conditions analysed. 
Therefore the conclusions reached about the effect of boundary conditions are robust also with respect to thickness.

In conclusion it has been shown that, when considering simplified models for sound radiation from plates, the effect of boundary conditions can play an important role. On average the radiation efficiency increases with an increasing amount of constraint at the edges but, in particular in the transition between the monopole region and the short circuiting region, some single third octave frequency bands can show the opposite effect and the correct result has to be assessed case by case. Examples of a less constrained plate radiating more than a more constrained one were already observed in the literature for the case of single-mode radiation efficiencies (see e.g. [6]). Results shown in this paper confirm this in the case of averaged radiation efficiency. Deeper investigations may be necessary in order to obtain complete conclusions on this point.

\section{Acknowledgements}

The authors wish to acknowledge the Italian University Catholic Centre (CUC) for having funded part of the research through a fellowship supporting the first author between 2011 and 2013.

They are also grateful to Prof. S. Miccoli for his valuable suggestions in software implementation and to J. V. Venini for his contribution during his master thesis.

\section{References}

[1] G. Xie, D.J. Thompson, C.J.C. Jones, The radiation efficiency of baffled plates and strips, Journal of Sound and Vibration 280 (2005) 181-209.

[2] J.W. Rayleigh, The Theory of Sound, 2nd Edition, Dover, 1945.

[3] G. Maidanik, Response of ribbed panels to reverberant acoustic fields, Journal of the Acoustical Society of America 34 (1962) 809-826.

[4] C.E. Wallace, Radiation resistance of a rectangular panel, Journal of the Acoustical Society of America 51 (1972) 946-952. 
[5] M.C. Gomperts, Radiation from rigid baffled, rectangular plates with general boundary conditions, Acustica 30 (1974) 320-327.

[6] M.C. Gomperts, Sound radiation from baffled, thin, rectangular plates, Acustica 37 (1977) 93102.

[7] M. Heckl, Radiation from plane sound sources, Acustica 37 (1977) 155-166.

[8] E.G. Williams, A series expansion of the acoustic power radiated from planar sources, Journal of the Acoustical Society of America 73 (1983) 1520-1524.

[9] F.G Leppington, E.G. Broadbent, K.H. Heron, The acoustic radiation efficiency of rectangular panels, Proceedings of the Royal Society of London A 382 (1982) 245-271.

[10] F.G. Leppington, E.G. Broadbent, K.H. Heron, Acoustic radiation from rectangular panels with constrained edges, Proceedings of the Royal Society of London. A. 393 (1984) 67-84.

[11] R.F. Keltie, H. Peng, The effects of modal coupling on the acoustic power radiation from panels, Journal of Vibration Acoustics Stress and Reliability in Design 109 (1987) 48-55.

[12] S.D. Snyder, N. Tanaka, Calculating total acoustic power output using modal radiation efficiencies, Journal of the Acoustical Society of America 97 (1995) 1702-1709.

[13] W.L. Li, H.J Gibeling, Determination of the mutual radiation resistance of a rectangular plate and their impact on the radiated sound power, Journal of Sound and Vibration 229 (2000) 12131233.

[14] W.L. Li, H.J Gibeling, Acoustic radiation from a rectangular plate reinforced by springs at arbitrary locations, Journal of Sound and Vibration 220 (1999) 117-133.

[15] J. Yoo, Study on the general characteristics of the sound radiation of a rectangular plate with different boundary edge conditions, Journal of Mechanical Science and Technology 24 (2010) 1111-1118. 
[16] A. Putra, D.J.Thompson, Sound radiation from rectangular baffled and unbaffled plates, Applied Acoustics 71 (2010) 1113-1125.

[17] A. Berry, J.L. Guyader, J. Nicolas, A general formulation for the sound radiation from rectangular, baffled plates with arbitrary boundary conditions, Journal of the Acoustical Society of America 88 (1990) 2792-2802.

[18] M.P. Norton, Fundamentals of Noise and Vibration Analysis for Engineers, Cambridge University Press, 1990.

[19] H. Nelisse, O. Beslin, J. Nicolas, A generalized approach for the acoustic radiation from baffled or baffled plate with arbitrary boundary condition, Journal of Sound and Vibration 211 (1998) 207-225.

[20] Y. Qiao, Q. Huang, L. Li, Influence of boundary conditions on sound radiation characteristic from rectangular plates, Low Frequency Noise \& Vibration and Active Control 26 (2007) 115133.

[21] X. Zhang, W. Li, L. Wen, A unified approach for predicting sound radiation from baffled rectangular plates with arbitrary boundary conditions, Journal of Sound and Vibration 329 (2010) 5307-5320.

[22] E. G. Williams, Numerical evaluation of the radiation from unbaffled, finite plates using the FFT, Journal of the Acoustical Society of America 74 (1983) 343-347.

[23] N. Atalla, J. Nicolas, and C. Gauthier, Acoustic radiation of an unbaffled vibrating plate with general elastic boundary conditions, Journal of the Acoustical Society of America 99 (1996) 14841494.

[24] C. H. Oppenheimer and S. Dubowsky, A radiation efficiency for unbaffled plates with experimental validation, Journal of Sound and Vibration 199 (1997) 473-489. 
[25] B. Laulagnet, Sound radiation by a simply supported unbaffled plate, Journal of the Acoustical Society of America 103 (1998) 2451-2462.

[26] A. Leissa, Vibration of Plates, ASOA, 1993.

[27] G.B. Warburton, The vibration of rectangular plates, Proceedings of the Institution of Mechanical Engineers 168 (1954) 371-384.

[28] A. W. Leissa, The free vibration of rectangular plates, Journal of Sound and Vibration 31(1973) 257-293.

[29] F. Fahy and J. G. Walker, Fundamentals of Noise and Vibration. Taylor and Francis, 1998 
Table 1. Beam functions for $x$ direction, [28] (S: simply supported edge, C: clamped edge, F: free edge).

$x=0 \quad x=\mathrm{a} \quad X(x)$

$\mathrm{S} \quad \mathrm{S} \quad \sin \frac{(m-1) \pi x}{a} \quad m=2,3,4, \ldots$

C C

$$
\begin{cases}\cos \gamma_{1}\left(\frac{x}{a}-\frac{1}{2}\right)+\frac{\sin \left(\gamma_{1} / 2\right)}{\sinh \left(\gamma_{1} / 2\right)} \cosh \gamma_{1}\left(\frac{x}{a}-\frac{1}{2}\right) & m=2,4,6, \ldots \\ \sin \gamma_{2}\left(\frac{x}{a}-\frac{1}{2}\right)-\frac{\sin \left(\gamma_{2} / 2\right)}{\sinh \left(\gamma_{2} / 2\right)} \sinh \gamma_{2}\left(\frac{x}{a}-\frac{1}{2}\right) & m=3,5,7, \ldots\end{cases}
$$

with $\gamma_{1}$ and $\gamma_{2}$ calculated as the roots of:

$$
\left\{\begin{array}{l}
\tan \left(\gamma_{1} / 2\right)+\tanh \left(\gamma_{1} / 2\right)=0 \\
\tan \left(\gamma_{2} / 2\right)-\tanh \left(\gamma_{2} / 2\right)=0
\end{array}\right.
$$

$$
\mathrm{F} \quad \mathrm{F}\left\{\begin{array}{l}
1 \quad m=0 \\
1-\frac{2 x}{a} \quad m=1 \\
\cos \gamma_{1}\left(\frac{x}{a}-\frac{1}{2}\right)-\frac{\sin \left(\gamma_{1} / 2\right)}{\sinh \left(\gamma_{1} / 2\right)} \cosh \gamma_{1}\left(\frac{x}{a}-\frac{1}{2}\right) \quad m=2,4,6, \ldots \\
\sin \gamma_{2}\left(\frac{x}{a}-\frac{1}{2}\right)-\frac{\sin \left(\gamma_{2} / 2\right)}{\sinh \left(\gamma_{2} / 2\right)} \sinh \gamma_{2}\left(\frac{x}{a}-\frac{1}{2}\right) \quad m=3,5,7, \ldots
\end{array}\right.
$$

with $\gamma_{1}$ and $\gamma_{2}$ calculated as for CC condition.

$$
\text { C } \quad \mathrm{S} \quad \sin \gamma_{2}\left(\frac{x}{2 a}-\frac{1}{2}\right)-\frac{\sin \left(\gamma_{2} / 2\right)}{\sinh \left(\gamma_{2} / 2\right)} \sinh \gamma_{2}\left(\frac{x}{2 a}-\frac{1}{2}\right) \quad m=2,3,4, \ldots
$$

with $\gamma_{2}$ calculated as for $\mathrm{CC}$ condition.

$$
1-\frac{x}{a} \quad m=1
$$

F $\quad \mathrm{S} \quad \sin \gamma_{2}\left(\frac{x}{2 a}-\frac{1}{2}\right)-\frac{\sin \left(\gamma_{2} / 2\right)}{\sinh \left(\gamma_{2} / 2\right)} \sinh \gamma_{2}\left(\frac{x}{2 a}-\frac{1}{2}\right) \quad m=2,3,4, \ldots$

$$
\begin{aligned}
& \text { C } \quad \mathrm{F} \quad \cos \frac{\gamma_{3} x}{a}-\cosh \frac{\gamma_{3} x}{a}+\left(\frac{\sin \gamma_{3}-\sinh \gamma_{3}}{\cos \gamma_{3}-\cosh \gamma_{3}}\right)\left(\sin \frac{\gamma_{3} x}{a}-\sinh \frac{\gamma_{3} x}{a}\right) \\
& m=1,2,3, \ldots
\end{aligned}
$$


with $\cos \gamma_{3} \cosh \gamma_{3}=-1$

Table 2. Combinations of boundary conditions considered within the paper for aspect ratio equal to 1.2 and corresponding natural frequencies of the first vibration modes (aluminium plate, $a=0.5 \mathrm{~m}$, $\mathrm{b}=0.6 \mathrm{~m}, \mathrm{~h}=3 \mathrm{~mm})$. The table includes the assumed loss factor values.

\begin{tabular}{cccccccccccc}
\hline$x=0$ & $x=\mathrm{a}$ & $y=0$ & $y=\mathrm{b}$ & & Loss factor & $\begin{array}{c}f_{1} \\
(\mathrm{~Hz})\end{array}$ & $\begin{array}{c}f_{2} \\
(\mathrm{~Hz})\end{array}$ & $\begin{array}{c}f_{3} \\
(\mathrm{~Hz})\end{array}$ & $\begin{array}{c}f_{4} \\
(\mathrm{~Hz})\end{array}$ \\
\hline $\mathrm{S}$ & $\mathrm{S}$ & $\mathrm{S}$ & $\mathrm{S}$ & 0.3 & 0.1 & 0.03 & 0.01 & 49.6 & 110.5 & 137.3 & 198.3 \\
\hline $\mathrm{C}$ & $\mathrm{C}$ & $\mathrm{C}$ & $\mathrm{C}$ & 0.3 & 0.1 & 0.03 & 0.01 & 91.5 & 165.6 & 206.0 & 275.5 \\
\hline F & F & F & F & & 0.01 & & $\begin{array}{c}35.1 \\
\left(4^{\text {th }}\right)\end{array}$ & $\begin{array}{c}46.1 \\
\left(5^{\text {th }}\right)\end{array}$ & $\begin{array}{c}66.3 \\
\left(6^{\text {th }}\right)\end{array}$ & $\begin{array}{c}84.8 \\
\left(7^{\text {th }}\right)\end{array}$ \\
\hline S & S & F & F & & 0.01 & & 29.3 & 43.2 & 87.2 & 117.0 \\
\hline F & F & C & C & & 0.01 & & 66.3 & 75.3 & 110.8 & 182.8 \\
\hline S & S & C & C & & 0.01 & & 66.8 & 147.5 & 150.0 & 227.9 \\
\hline C & F & C & F & & 0.01 & & 18.7 & 58.4 & 74.6 & 126.2 \\
\hline C & S & C & S & 0.01 & & 68.6 & 136.7 & 170.1 & 234.4 \\
\hline F & S & F & S & & 0.01 & & 8.8 & 41.3 & 52.8 & 99.5 \\
\hline
\end{tabular}

Table 3. Plate geometry and first natural frequencies for different aspect ratios.

\begin{tabular}{ccccccccc}
\hline$a(\mathrm{~m})$ & $b(\mathrm{~m})$ & $h(\mathrm{~m})$ & Aspect ratio & $f_{1, S S}(\mathrm{~Hz})$ & $f_{2, S S}(\mathrm{~Hz})$ & $f_{3, S S}(\mathrm{~Hz})$ & $f_{4, S S}(\mathrm{~Hz})$ & $f_{c}(\mathrm{kHz})$ \\
\hline 0.5 & 0.6 & 0.003 & 1.2 & 49.6 & 110.5 & 137.3 & 198.3 & 4.02 \\
\hline 0.42 & 0.93 & 0.003 & 2.2 & 49.9 & 75.3 & 117.5 & 174.3 & 4.02 \\
\hline 0.4 & 1.4 & 0.003 & 3.5 & 49.4 & 60.6 & 79.3 & 105.4 & 4.02 \\
\hline
\end{tabular}

Table4. Plate geometry and first natural frequencies for different thicknesses values.

\begin{tabular}{ccccccccc}
\hline$a(\mathrm{~m})$ & $b(\mathrm{~m})$ & $h(\mathrm{~m})$ & Aspect ratio & $f_{1, S S}(\mathrm{~Hz})$ & $f_{2, S S}(\mathrm{~Hz})$ & $f_{3, S S}(\mathrm{~Hz})$ & $f_{4, S S}(\mathrm{~Hz})$ & $f_{c}(\mathrm{kHz})$ \\
\hline 0.5 & 0.6 & 0.002 & 1.2 & 33.0 & 73.7 & 91.5 & 132.2 & 6.02 \\
\hline 0.5 & 0.6 & 0.003 & 1.2 & 49.6 & 110.5 & 137.3 & 198.3 & 4.02 \\
\hline 0.5 & 0.6 & 0.005 & 1.2 & 82.6 & 184.2 & 228.9 & 330.4 & 2.41 \\
\hline
\end{tabular}




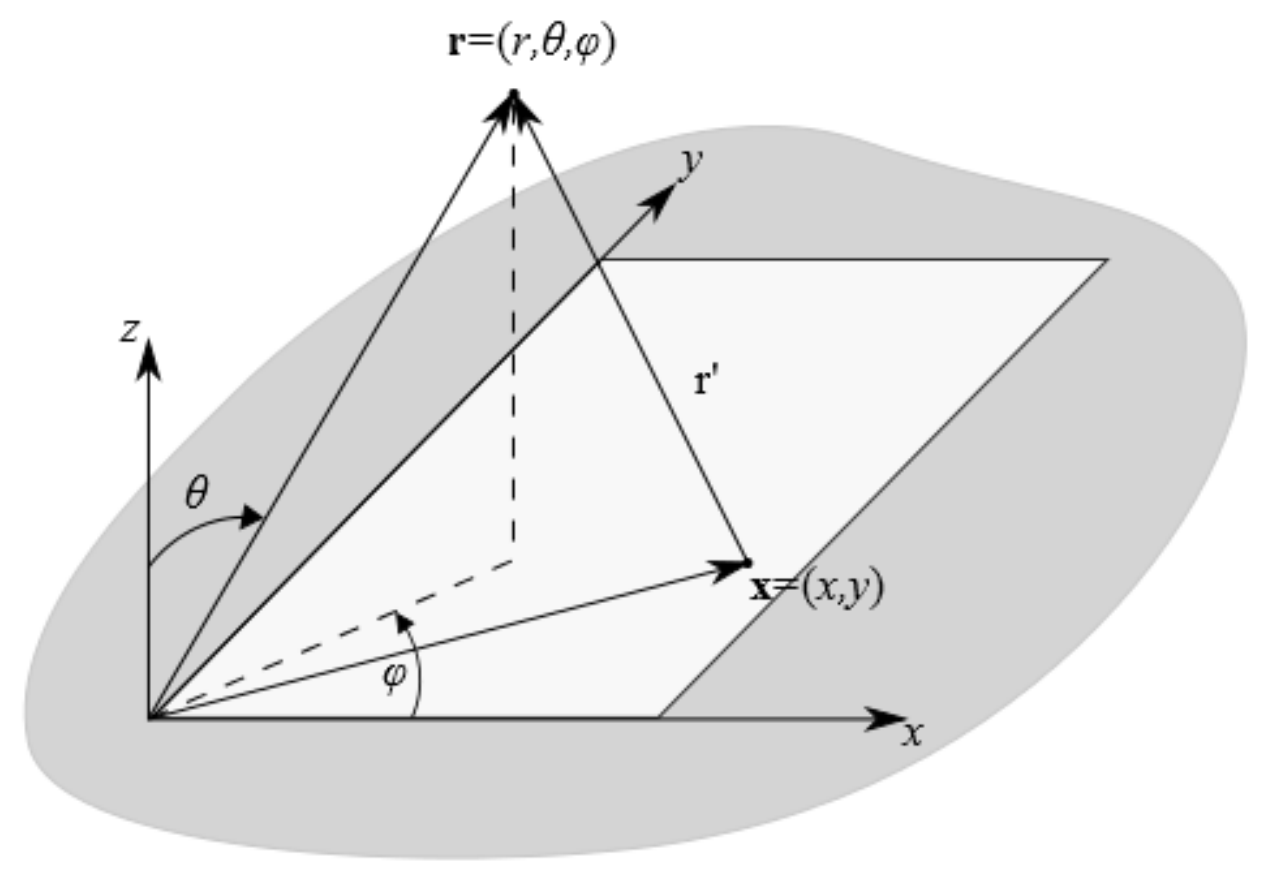

Figure 1. Rectangular plate set in an infinite rigid baffle.

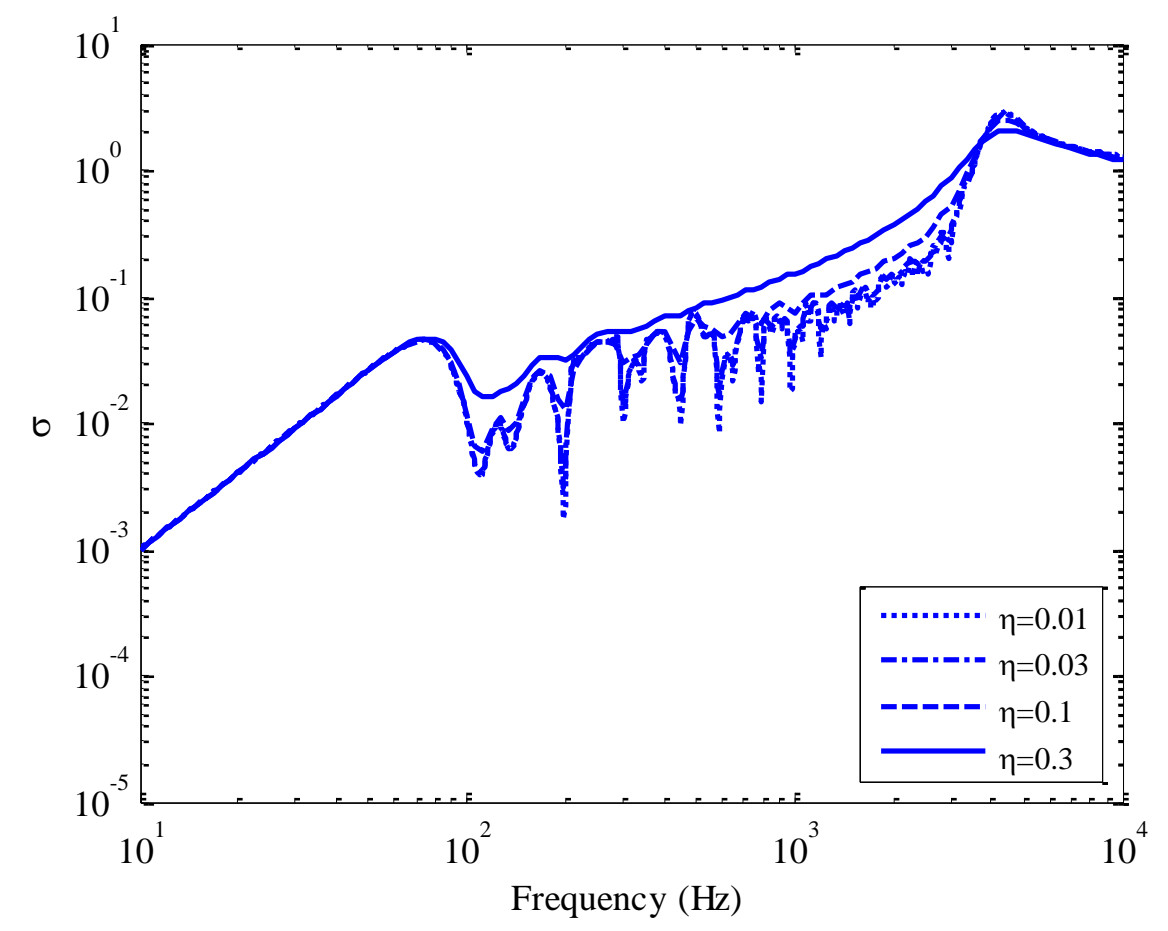

Figure 2. Average radiation efficiency of simply supported plate (Table 2) for different damping values. 


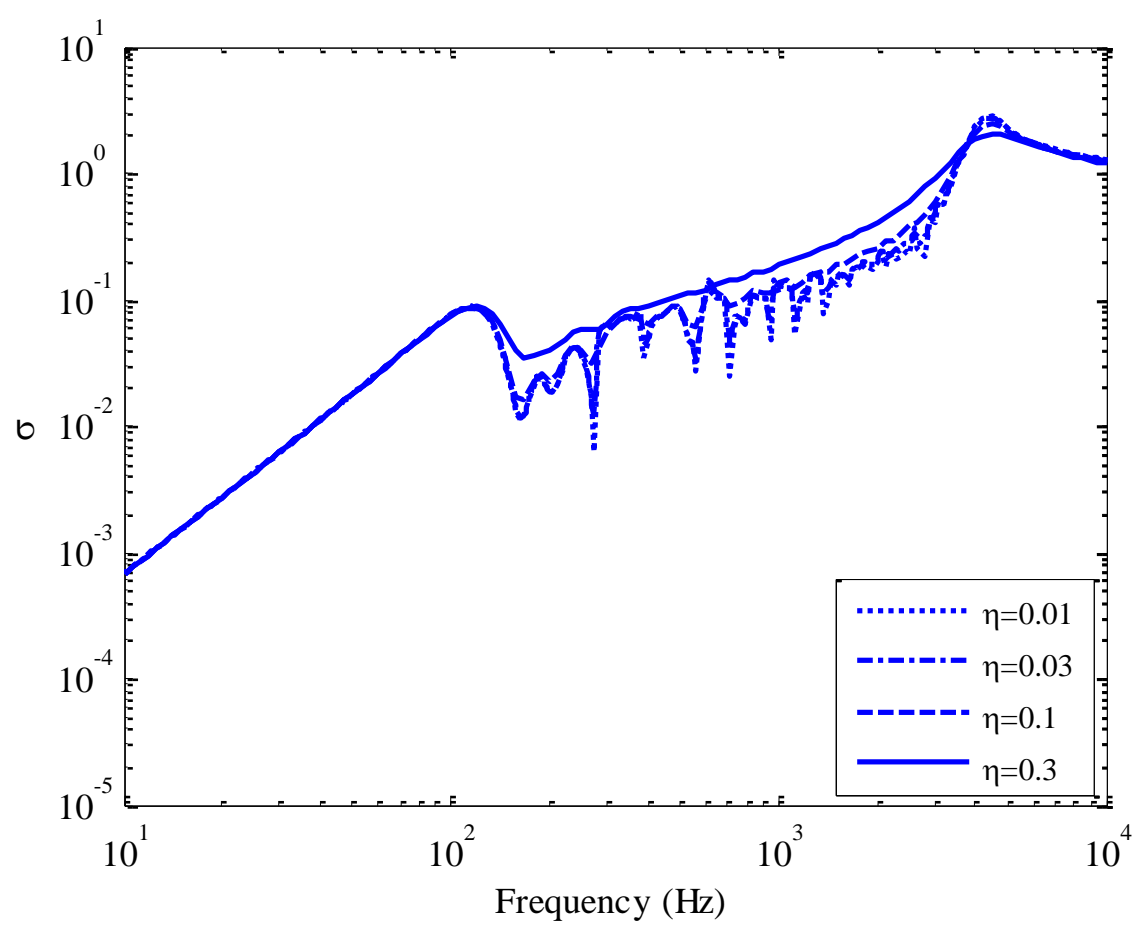

Figure 3. Average radiation efficiency of plate with fully clamped edges (Table 2) for different damping values.
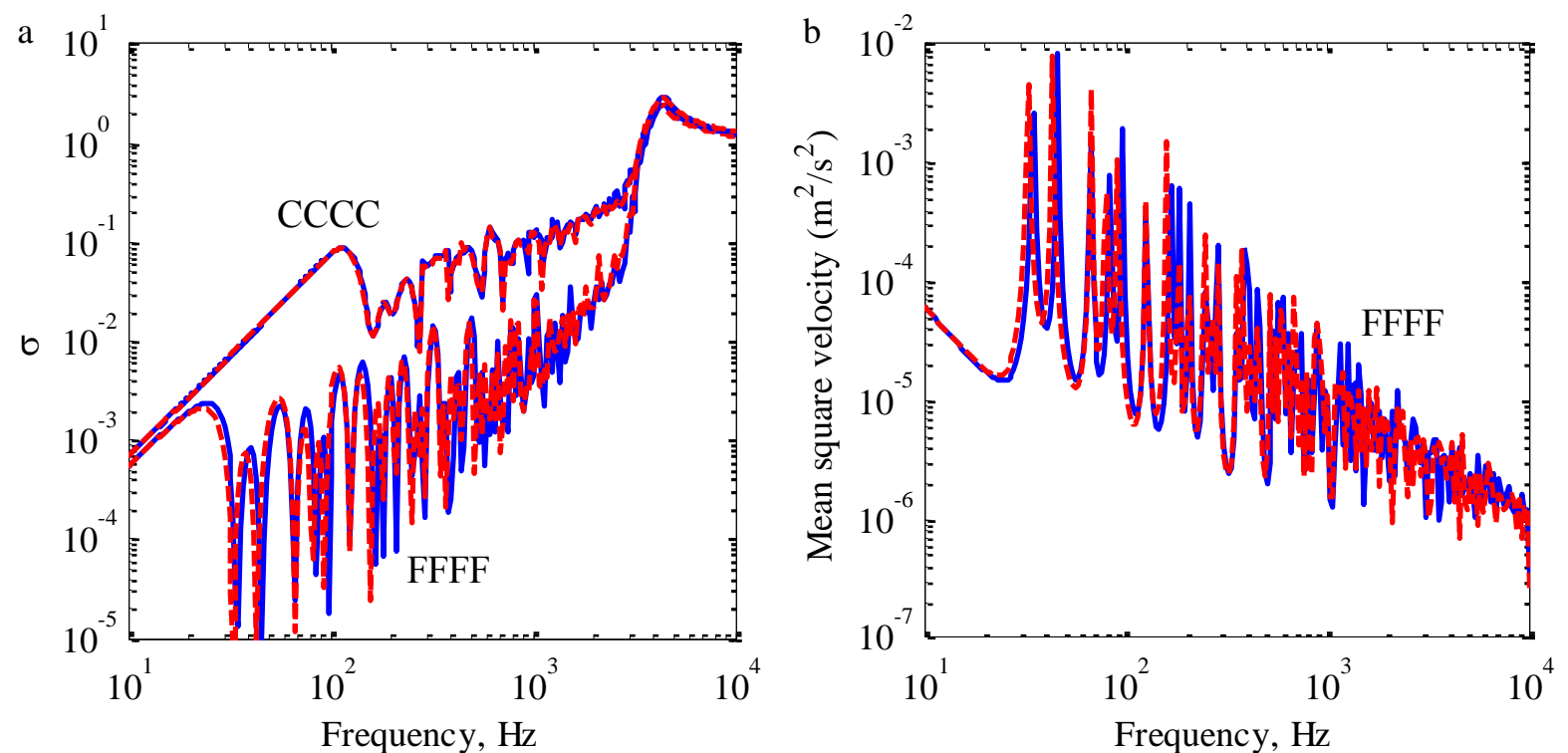
Figure 4: Results obtained by adopting analytical (continuous line) and numerical (FE) modal basis (dashed line). (a) Radiation efficiency for fully free and fully clamped plate (Table 2). (b) Mean square velocity for fully free plate.

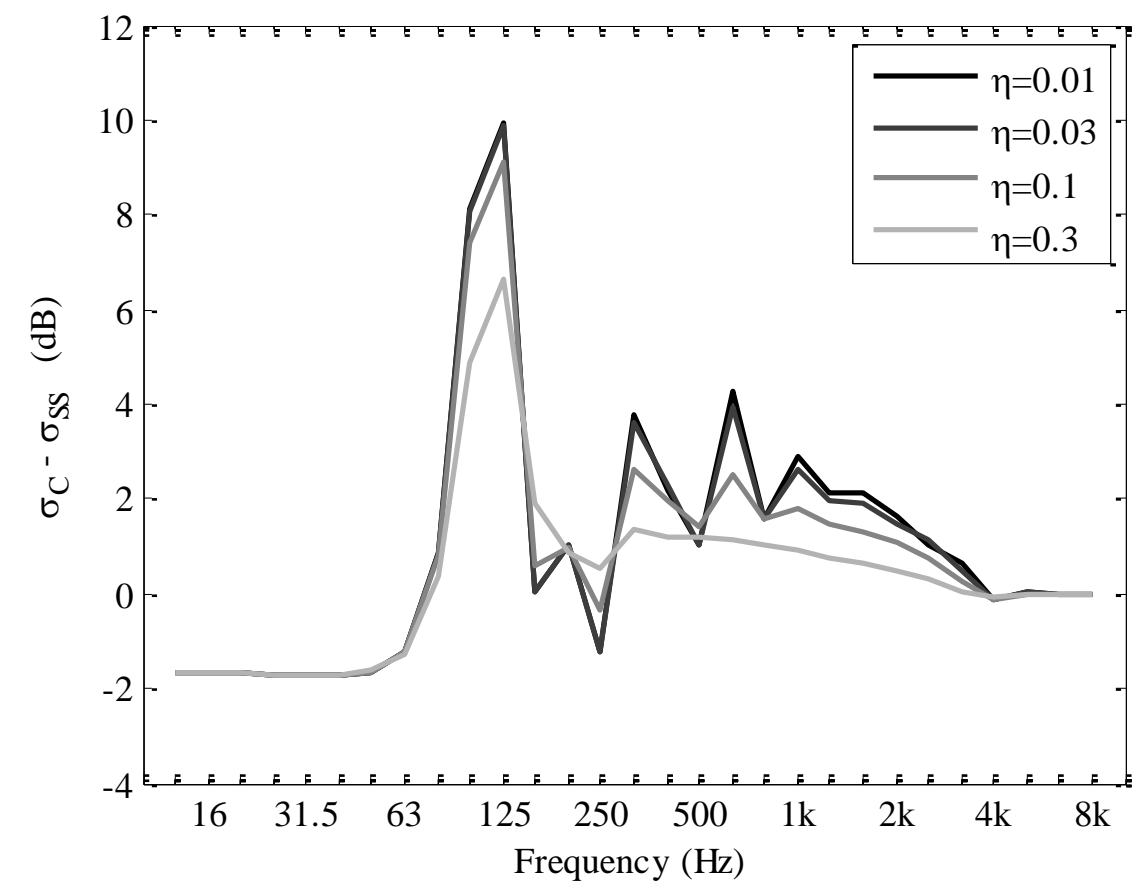

Figure 5. Third octave band difference between simply supported and clamped plate
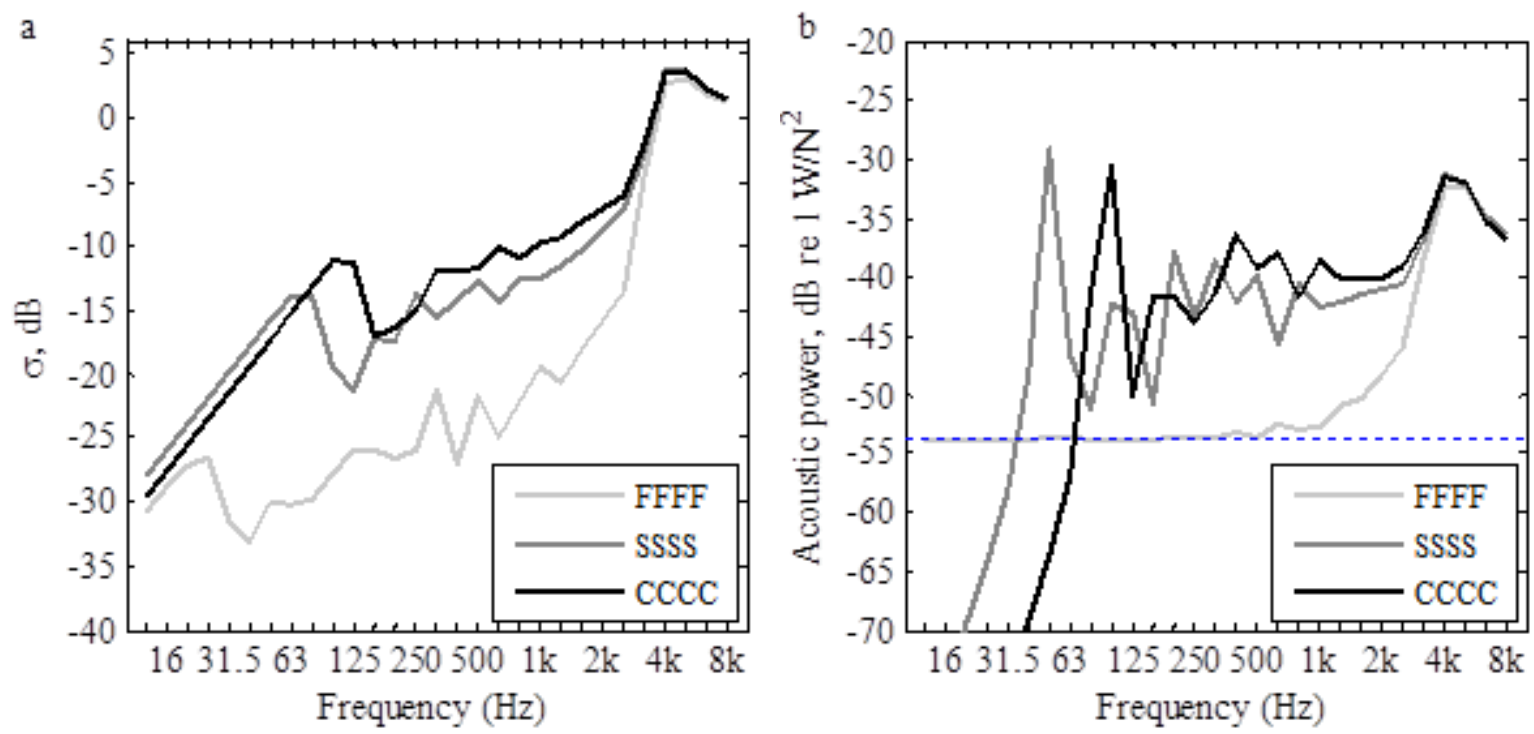
Figure 6. Plates having the same boundary conditions along the four edges. a: average radiation efficiency: b: acoustic power per unit force squared. Broken line: acoustic power of an equivalent rigid piston.
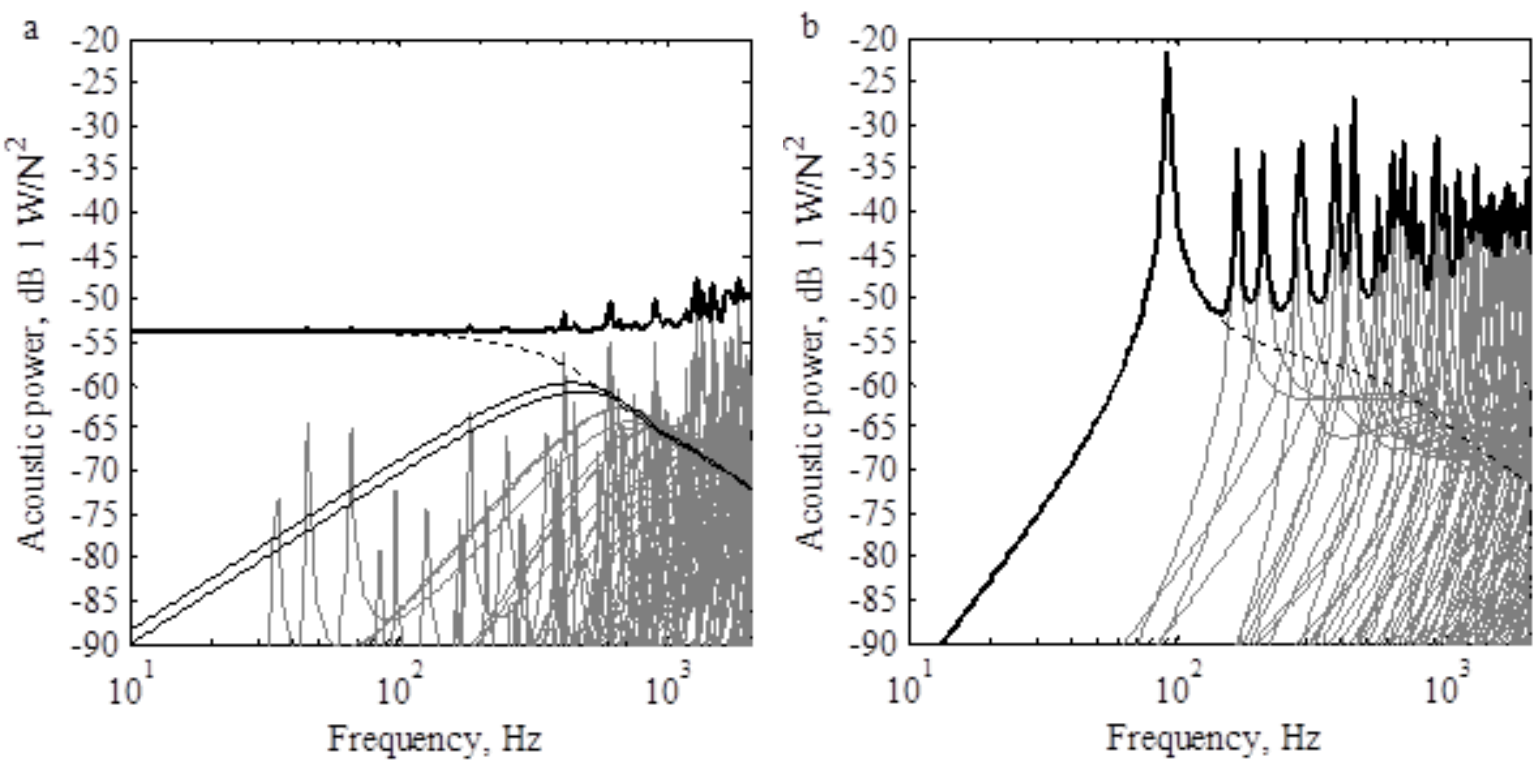

Figure 7. Narrow band plot of acoustic power. Thick dark line: total power; thin lines: single mode contribution; dashed line: acoustic power due to the first mode. a: FFFF plate; b: CCCC plate.
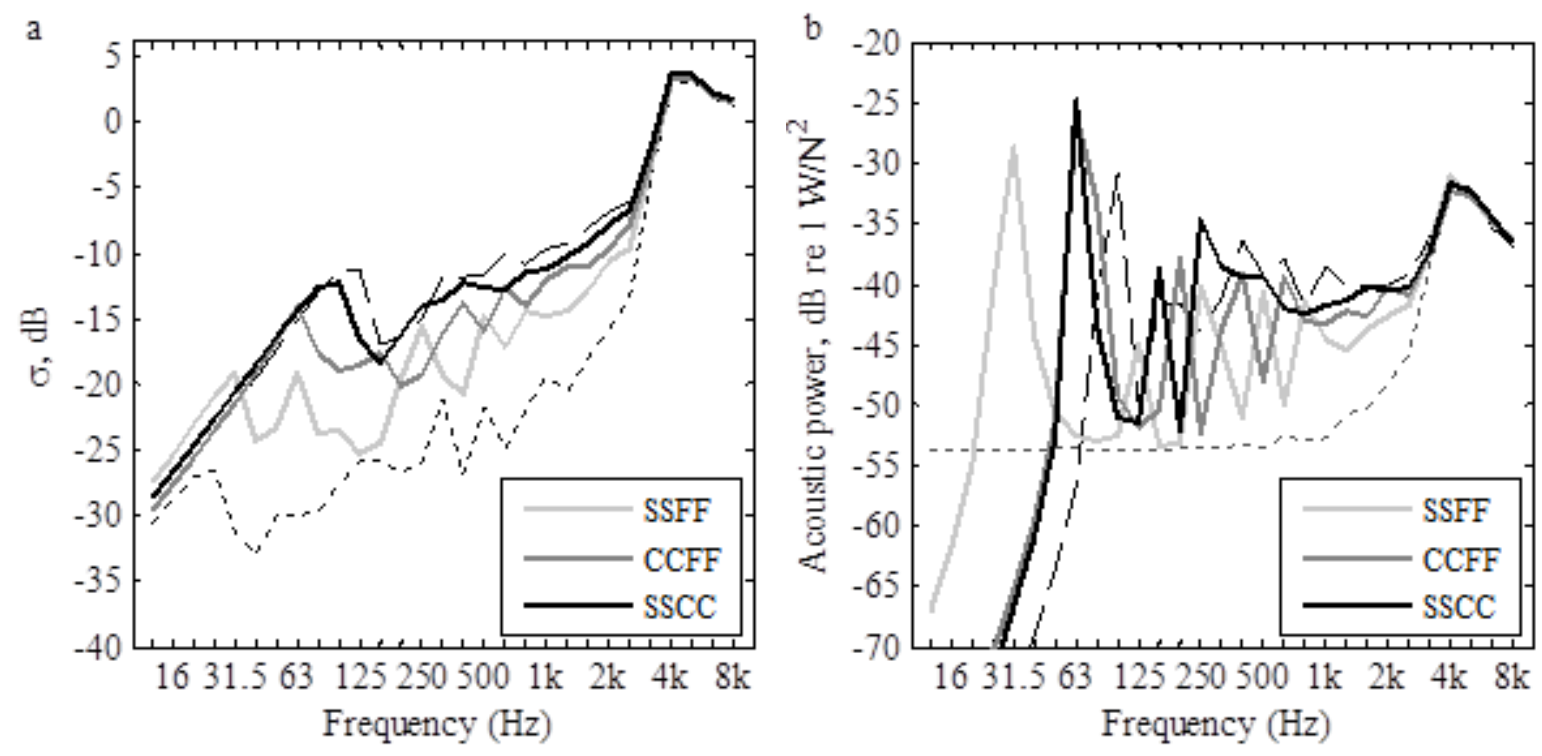

Figure 8. Plates having the same boundary condition on opposite edges. a: radiation efficiency: b: acoustic power per unit force squared. FFFF and CCCC are shown as broken lines for reference. 

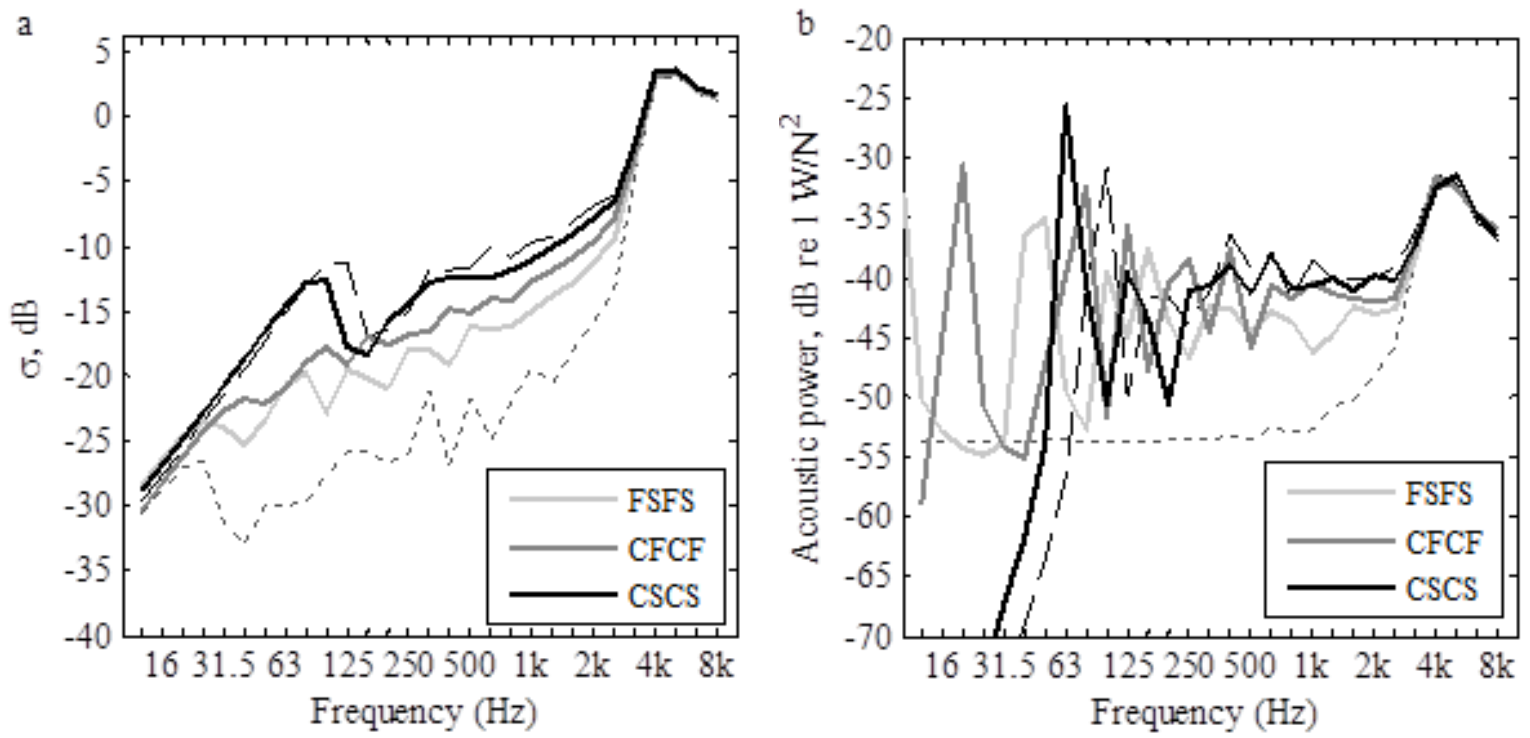

Figure 9. Plates having different boundary conditions on opposite edges. a: radiation efficiency: b: acoustic power per unit force squared. FFFF and CCCC are shown as broken lines for reference. 

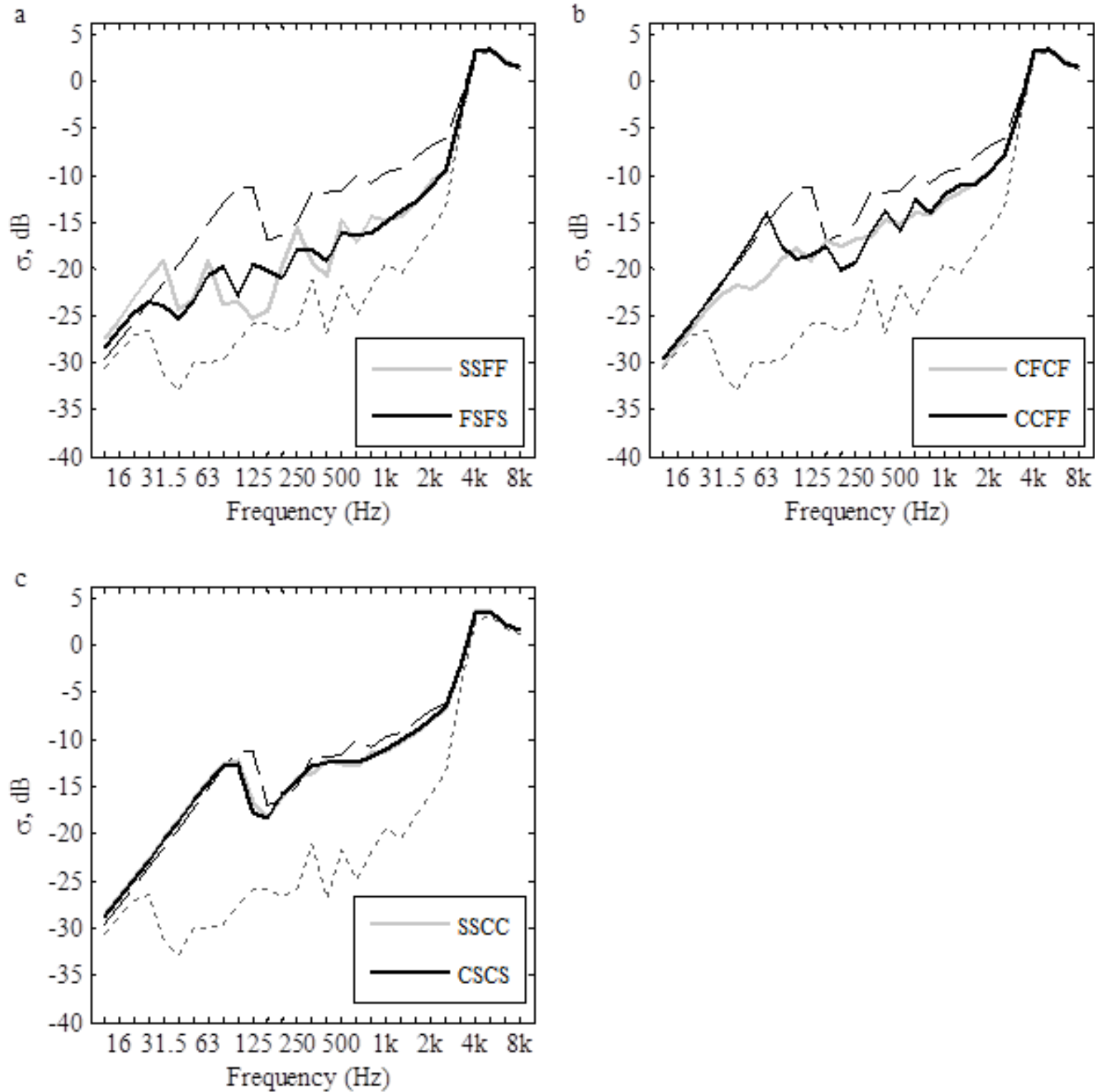

Figure 10. Radiation efficiencies for pairs of boundary conditions applied to different edges. a: combination of S-F; b: combinations of C-F; c: combinations of C-S. FFFF and CCCC are shown as broken lines for reference. 

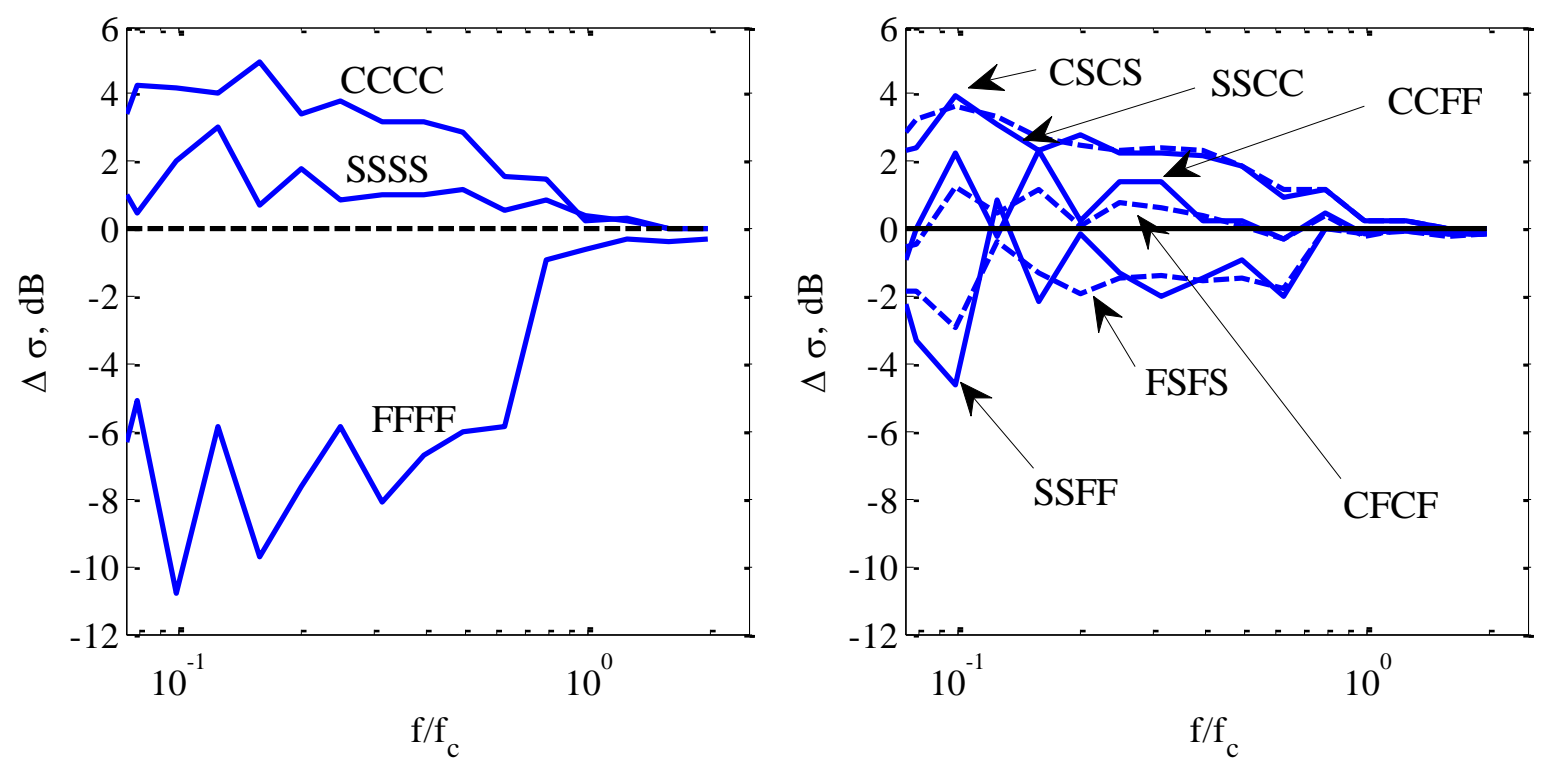

Figure 11: Radiation efficiencies normalised to Maidanik formula [3] and to critical frequency for various combination of boundary conditions.
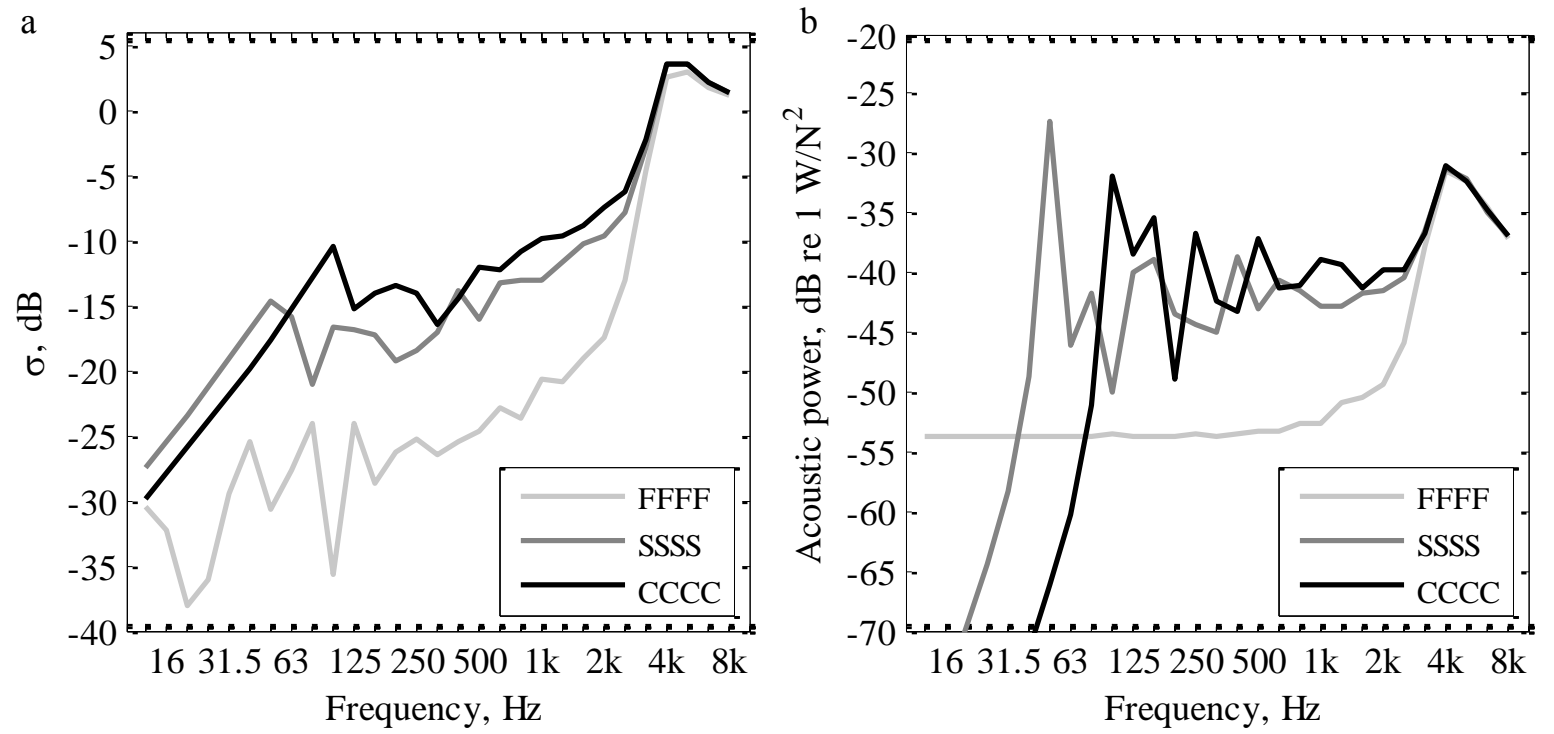

Figure 12. Plate with aspect ratio 2.2. a: radiation efficiency: b: acoustic power per unit force squared. 

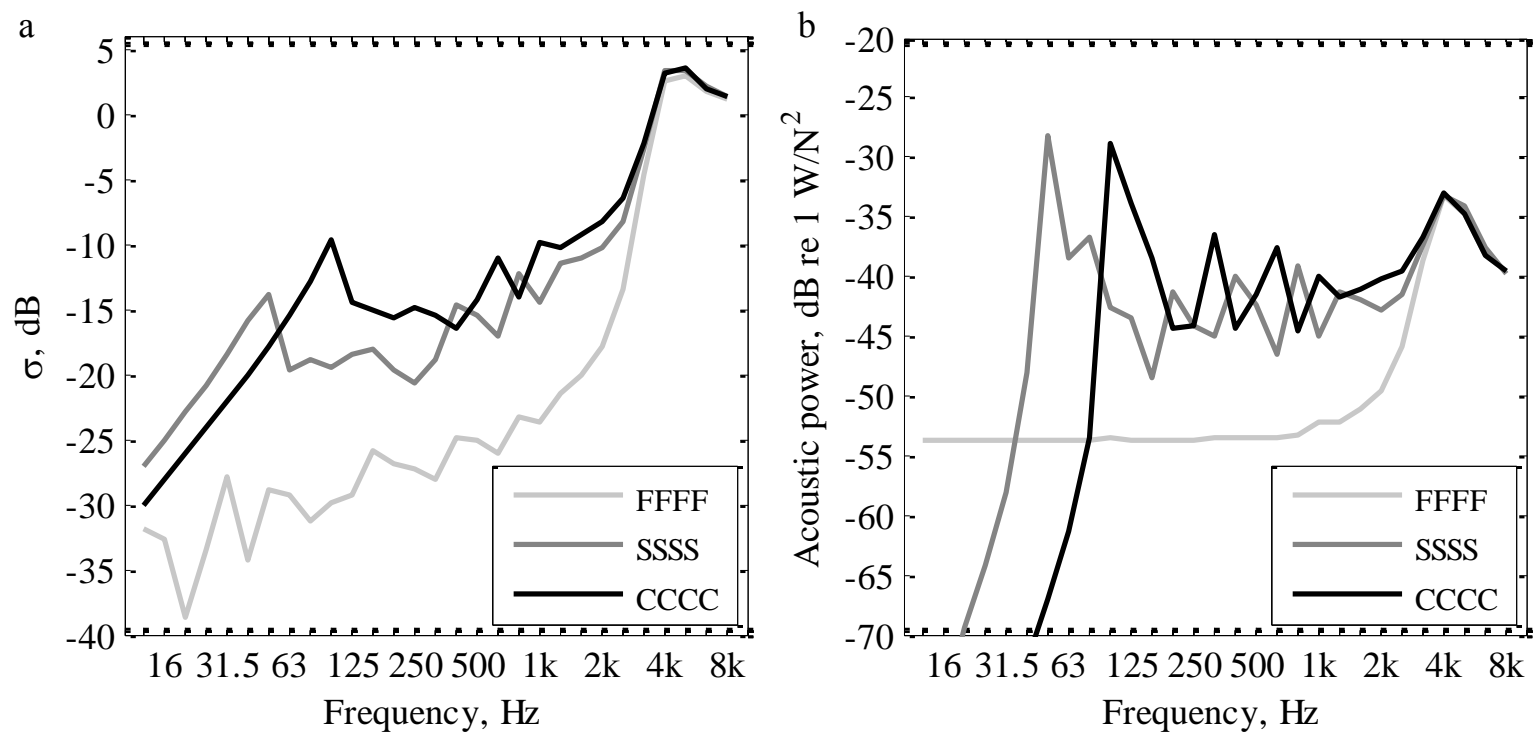

Figure 13. Plate with aspect ratio 3.5. a: radiation efficiency: b: acoustic power per unit force squared.

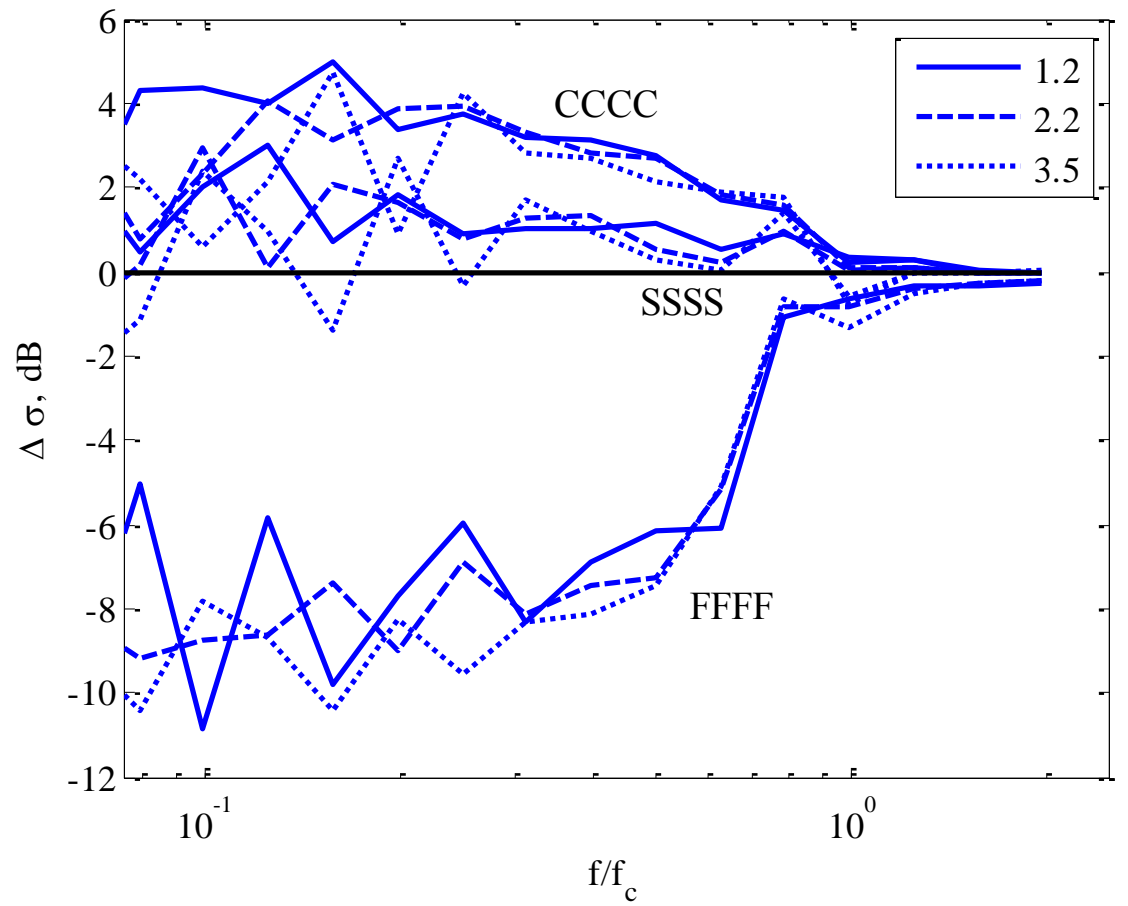

Figure 14. Radiation efficiencies normalised to Maidanik formula [3] and to critical frequency for three aspect ratios (1.2, 2.2 and 3.5) and for three different boundary conditions (FFFF, SSSS and CCCC). 

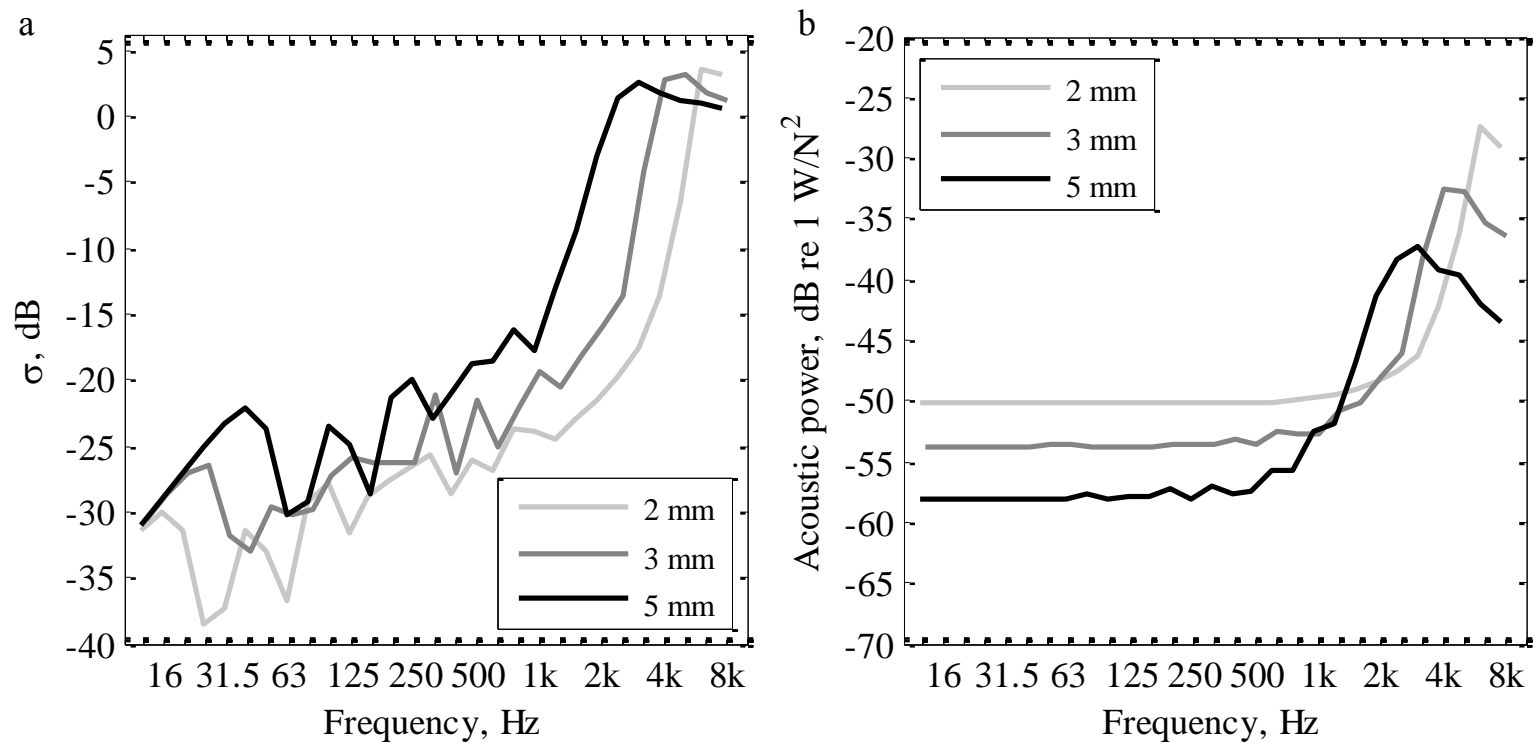

Figure 15. FFFF plate, effect of thickness (Table 4): a: average radiation efficiency; b: Acoustic power per unit force squared.
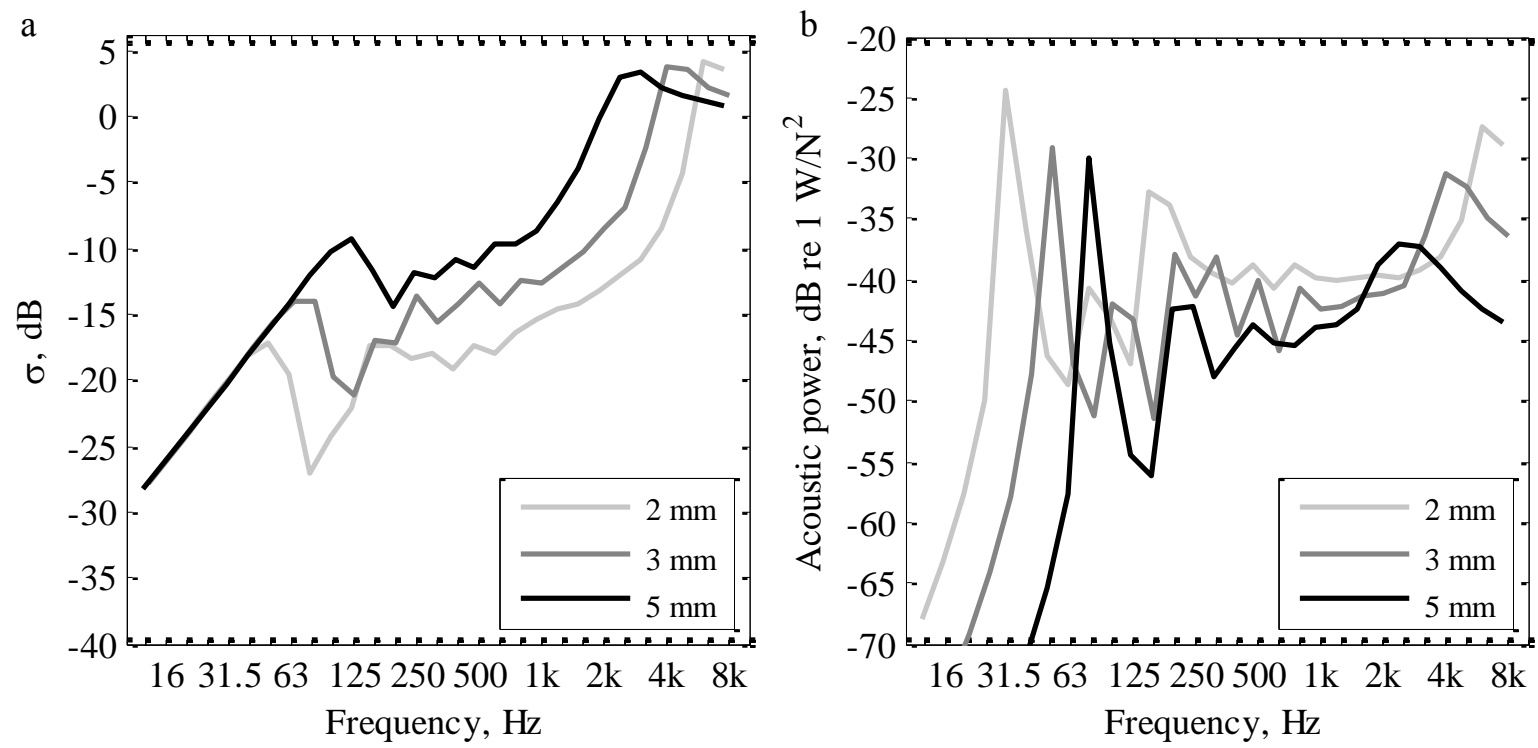

Figure 16. SSSS plate, effect of thickness (Table 4). a: average radiation efficiency; b: acoustic power per unit force squared. 

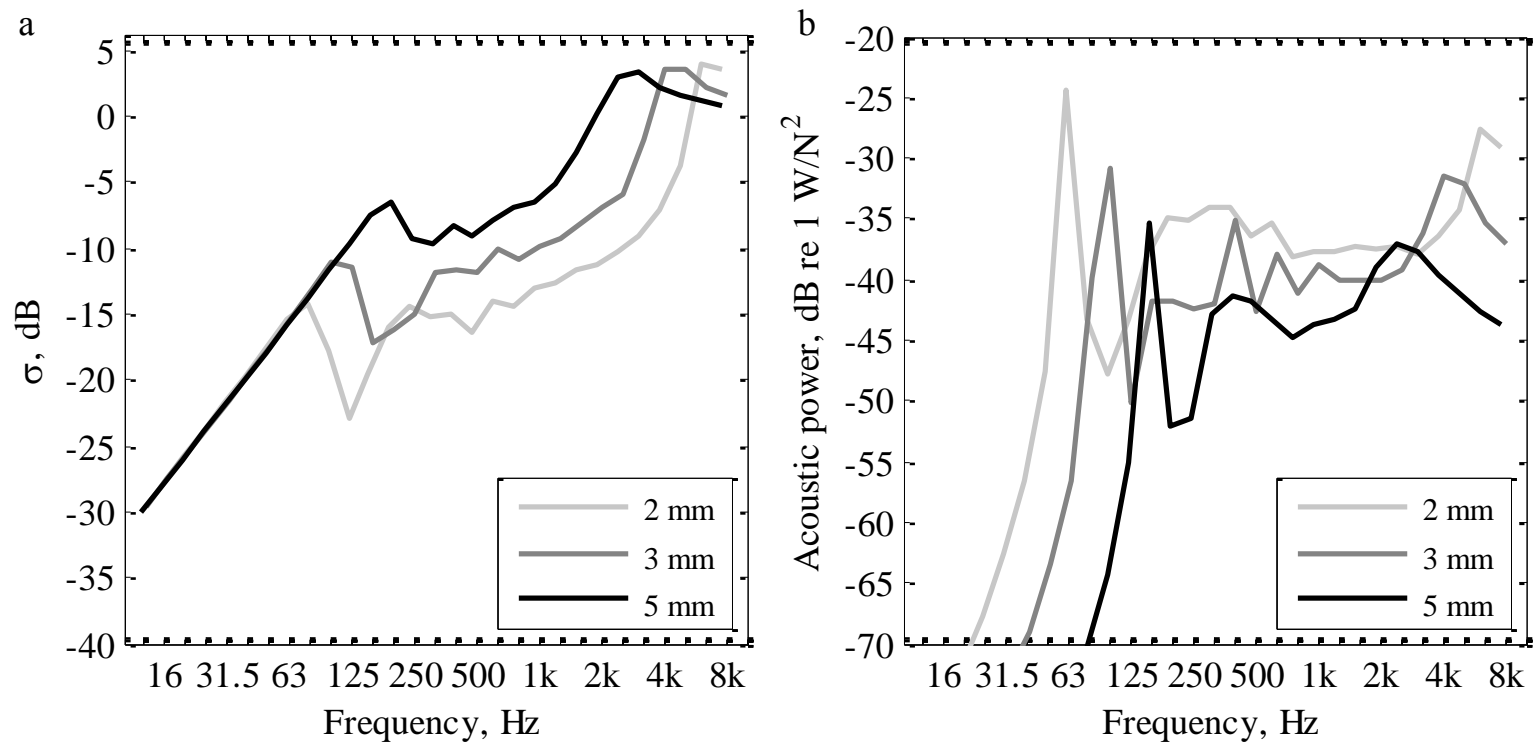

Figure 17.CCCC plate, effect of thickness (Table 4). a: average radiation efficiency; b: Acoustic power per unit force squared.

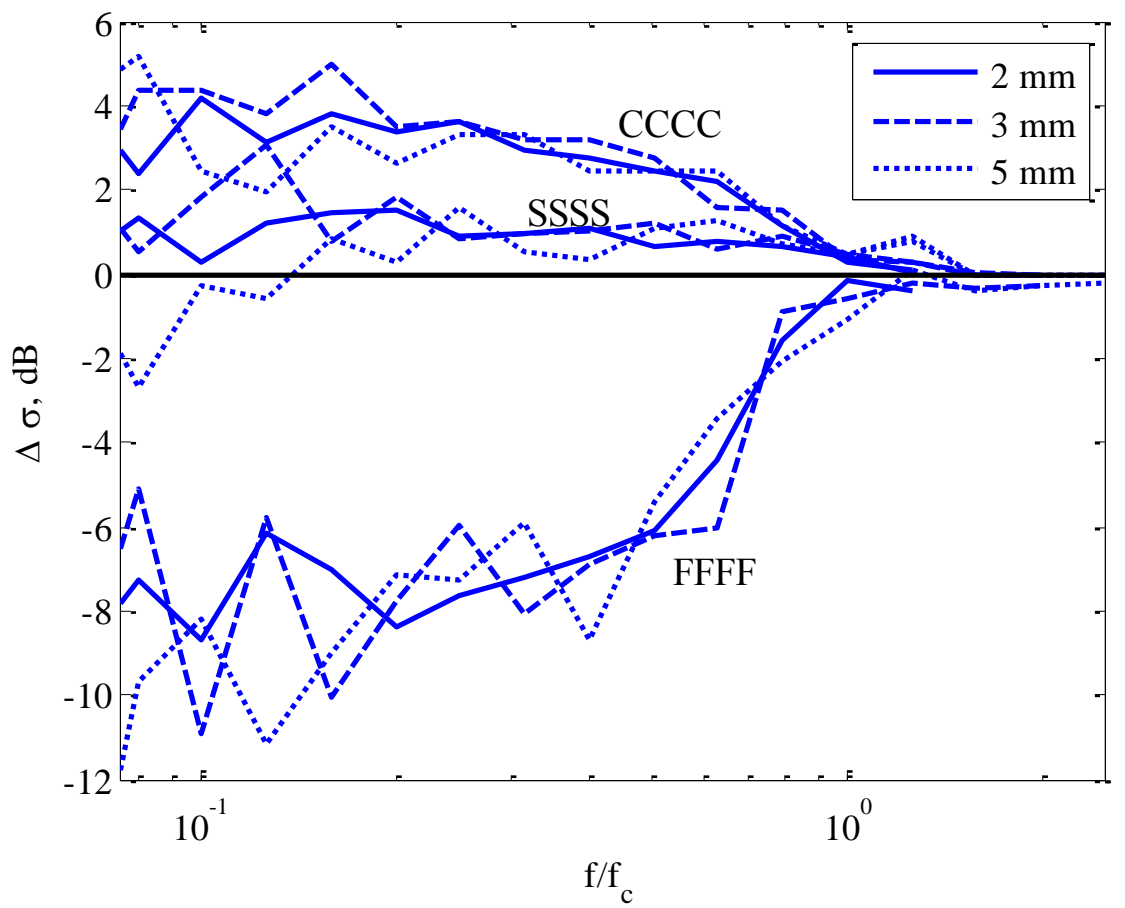

Figure 18. Radiation efficiencies normalised to Maidanik formula [3] and to critical frequency for three aspect thickness values ( $2 \mathrm{~mm}, 3 \mathrm{~mm}$ and $5 \mathrm{~mm}$ ) and for three different boundary conditions (FFFF, SSSS and CCCC). 
